\title{
Numerical Investigation of Influence of Nanoparticles Presence on Water Vapor Condensation Process inside a Vertical Channel
}

\author{
Mustapha Ait Hssain (D, Sara Armou, Kaoutar Zine-Dine, Rachid Mir, \\ and Youness El Hammami
}

Laboratory of Mechanics, Processes, Energy, and Environment (LMPEE) National School of Applied Sciences, Ibn Zohr University, Agadir, Morocco

Correspondence should be addressed to Mustapha Ait Hssain; ait.hssain90@gmail.com

Received 16 February 2021; Revised 5 April 2021; Accepted 27 April 2021; Published 17 May 2021

Academic Editor: P. Davide Cozzoli

Copyright (C) 2021 Mustapha Ait Hssain et al. This is an open access article distributed under the Creative Commons Attribution License, which permits unrestricted use, distribution, and reproduction in any medium, provided the original work is properly cited.

\begin{abstract}
This paper is aimed at investigating the nanofluid film condensation by mixed convection in the presence of water vapor, $\mathrm{Cu}$ nanoparticles, and air treated as a noncondensable gas (NCG) on the inner walls of a vertical channel. In this simulation, the flow is laminar, stationary, two dimensional, and axisymmetric. The coupled governing equations for the liquid film with the nanoparticles and the mixture air-humid-nanoparticles are solved together using the finite volume method. Since the application of humid air condensation is one of the most applicable methods of phase change processes that is observed in different industrial fields such as heating, ventilation, and air conditioning (HVAC) or cooling systems, for this purpose, the influence of injecting a uniform volume fraction of nanoparticles on improving heat and mass transfer is determined as a function of the variation in relative humidity, velocity, temperature, pressure, and volume fraction of $\mathrm{Cu}$ nanoparticles at the channel inlet. The numerical results indicate that under the best conditions in the range of variation studied $\mathrm{RH}_{\mathrm{in}}=100 \%, \mathrm{Re}_{\mathrm{in}}$ $=2000, T_{\text {in }}=50^{\circ} \mathrm{C}, P_{\text {in }}=0.5 \mathrm{~atm}$, and $\varphi_{\text {in }}=0.1 \%$, the use of nanoparticles has a greater impact, and the maximum improvement in the condensation film thickness, the local Nusselt number, and the accumulated condensation rate has an effective ratio strictly greater than one compared with the case of pure humid air.
\end{abstract}

\section{Introduction}

In this work, film condensation of moist air by mixed convection in a vertical channel in the presence of nanoparticles was numerically studied. Condensation of humid air is one of the most applicable phase change processes in various industrial equipment [1] such as ventilation, cooling, desalination, and heating devices. Indeed, various studies have focused on the improvement of film condensation of pure vapors or mixtures of vapor and NCG, by investigating the influence of parameters affecting this phenomenon such as $P_{\text {in }}, W_{\text {in }}, T_{\text {in }}$ , $\operatorname{Re}_{\text {in }}$, and $\Delta T$ in different geometries (horizontal, inclined, or vertical) using different numerical or experimental methods. A recent technique to enhance the heat and mass transfer is made by using solid nanoparticles in the base fluid. As a result, some researchers have attempted to evaluate the effect of nanoparticles on improving the heat and mass transfer process during condensation. Therefore, in-depth studies on the effects of adding nanoparticles in a base fluid on simultaneous heat and mass transfer with phase change are necessary to understand their influence on the condensation. The literature review is divided into three parts: the first part is about convective heat transfer enhancement using nanoparticles, the second part is about condensation without nanoparticles, and the last part is about condensation with suspended nanoparticles.

1.1. Nanoparticles Application in Convection Heat Transfer. The modeling of heat transfer enhancement with or without the addition of nanoparticles is important for several processes; many works in the literature have been focused on the study of the presence of a magnetic field or nanoparticles 
on heat transfer enhancement, among which are the following.

Mahian et al. [2] presented an analytical solution of the equations governing the entropy generation due to the mixed MHD convection between two isothermal cylinders bathed in a magnetic field. The results of this study show that the entropy generation decreases with the increase of the magnetic field. In this sense of MHD flows, the authors AlSalem et al. [3] have performed a numerical study on MHD convection in a cavity with a linearly heated bottom wall. The finite volume method was used to solve the basic equations. The results show that the heat transfer decreases with the increase of the magnetic field for all the studied parameters. Oztop et al. [4] published a study based on the finite volume method, in which they used a heat line visualization technique to understand the heat transport path in a nonuniformly heated inclined cavity filled with water-based $\mathrm{CuO}$ nanofluid. The results confirmed that the heat transfer in the cavity increases with the addition of nanoparticles. Shirkhani et al. [5] have analytically studied the problem of nonlinear fins with temperature dependent thermal conductivity and heat transfer coefficient using the collocation method $(\mathrm{CM})$, the variation iteration method (VIM), and the homotopy perturbation method (HPM). The results found reveal that the CM is very efficient, simple, and more accurate than the other techniques. Peiravi et al. [6] conducted a study on the effect of using multiphase nanofluids and the Rayleigh number on the heat transfer rate in a $3 \mathrm{D}$ enclosure using the Boltzmann multiphase thermal lattice. The results show that by choosing the appropriate height and arrangement of baffles, the average Nusselt number can be changed by more than 57\%. The same author, Peiravi et al. [7], published a paper on a three-dimensional heat transfer analysis for a partially heated enclosure led by a vertical fin mounted on the hot wall. These authors illustrated the effects of vertical fins and nanoparticles on the flow and on the thermal fields. The results show that the use of nanofluids $(\varphi=0.03$, Cuo/water) improves the heat transfer mechanism in the cubic enclosure.

1.2. Humid Air Condensation. The subject of film condensation has been widely studied since its first appearance with the work of Nusselt [8] in 1916. Several authors have inspired the Nusselt model to conduct more general and in-depth studies. Srzic et al. [9] conducted a computational study on the film condensation of a vapor along an inclined plate in the presence of light gases. Their model is established using the boundary layer equations for both phases that are solved by the finite volume method. Their results indicate that the reduction of heat and mass transfer in the presence of gases is all the more important when the molecular weight of the gas is low. Hammou et al. [10] studied numerically the mixed convection of moist air resulting from a laminar and twodimensional flow in a downward channel with isothermal wall and covered with a thin film of liquid water. Their results reveal that the enhancement in the temperature of the entrance zone causes a slight increase in the number of latent Nusselt. In addition, they observed that the velocity profiles, the coefficient of friction, and the Nusselt and the Sherwood numbers are remarkably influenced by Archimedes forces. Maheshwari et al. [11] carried out a numerical study of the influence of the Reynolds number on the condensed steam flow rate during the condensation of a mixture of vapor and NCG in a vertical tube. Their results show that, for the small Reynolds numbers at the inlet, the thermal resistance of the NCG boundary layer at the interface is greater than that of the condensate film. However, these results are reversed for elevated Reynolds numbers. Mohamed et al. [12] presented a model for the computational investigation of the condensation of humid air in the presence of liquid film flowing down inside a vertical channel. It was noticed that growing the quantity of water film or decreasing its inlet temperature promotes the condensation process.

In recent times, since 2011, several authors have been interested in the study of condensation under various conditions and different geometries. These studies include the following: Giri et al. [13], Li [14], Caruso et al. [15, 16], Zschaeck et al. [17], Hammoudi et al. [18], Hssain et al. [19], Wu et al. [20]. These authors carried out numerical studies of mixed and natural convection heat and mass transfers in different geometries and by different numerical methods. These models are based on the governing equations: continuity, momentum, energy, and diffusion. In these studies, several parameters were tested such as the inlet Reynolds numbers, the inlet pressure, the thermal Grashoff number, and the NCG fraction. Their main results showed that the decrease in the quantity of NCG favors the condensation of steam; in addition, the number of local sensitive Nusselt does not depend on the entry speed. However, the number of Nusselt due to condensation depends entirely on the flow rate.

1.3. Nanoparticles Application in Condensation. The number of publications devoted to the study of heat and mass transfer during condensation in the presence of nanoparticles remains rather limited, despite its importance in improving the efficiency of industrial processes. Nanofluids have become a new frontier for scientific research because of the higher thermophysical properties than those of the base fluid. Avramenko et al. [21,22] conducted a numerical study on heat and mass transfer during steam condensation in the presence of nanoparticles on a flat plate. In this simulation, the authors added an equation for nanoparticle concentration, which describes the increase in heat and mass transfer due to the presence of nanoparticles. This study shows that an increase in the fraction of nanoparticles leads to a significant improvement in heat and mass transfer. Turkyilmazoglu [23] modified the classical Nusselt model to simulate the condensation of nanofluid on a vertical wall. Five types of nanoparticles, namely $\mathrm{Ag}, \mathrm{Cu}, \mathrm{CuO}, \mathrm{Al}_{2} \mathrm{O}_{3}$, and $\mathrm{TiO}_{2}$, were considered. These authors showed that nanofluids improve the thermal transfer rate due to their excellent thermal conductivity. In addition, the thickness of the condensate film decreases with increasing heat transfer rate when a larger volume fraction of nanofluids is added. Mghari et al. [24] conducted a numerical study of heat and mass transfer during the condensation of a nanofluid in a horizontal channel. The equation system is solved by the Runge-Kutta method. 
The condensate film thickness is determined by the dichotomy method between the outlet and the inlet of the channel. Their results show that adding a $5 \%$ of $\mathrm{Cu}$ nanoparticles improves the thermal transfer coefficient by $20 \%$. They also found that the pressure drop increases by increasing the concentration of nanoparticles in the base fluid. Correlations have been proposed by these authors to predict the average coefficient of heat transfer during the condensation of nanofluids in a channel. These authors proposed correlations to predict the average coefficient of heat transfer during the condensation of nanofluids in a channel. Malvandi et al. [25] focused on the condensation of nanofluids into falling film by considering in their model the migration effects of nanoparticles in the liquid film. Two types of nanoparticles, titania and alumina, were studied to determine their effect on thermal transfer. The results show that as the diameter of the nanoparticles increases, their migration increases in the liquid film, increasing the volume fraction of the nanoparticles on the cold wall. Famileh et al. [1] numerically studied the influence of the addition of nanoparticles in the gas phase on the condensation of moist air. The equations governing heat and mass transfers in both phases were solved by the FLUENT software. Results show that an enhancement of mass fraction of nanoparticles has a direct influence on the condensate Reynolds number and humid air Sherwood number; these effects are more impressive for higher relative humidities. These results show also that under all conditions studied, the use of nanoparticles improved the rate of condensation, and the enhancement was not uniform for all inlet conditions. Hatami et al. [26] presented an analysis of the condensation of nanofluids in two phases between two vertical plates under the influence of magnetic and gravitational forces. They used the fourth-order Runge-Kutta numerical method to solve the equations governing heat and mass transfer in both phases. The results show that the thermophoretic parameter and viscosity parameter have significant effects on temperature and nanoparticles concentration profiles as well as on the Nusselt number. Peng et al. [27] conducted an experimental analysis of R141b condensation in the presence of $\mathrm{CuO}$ nanoparticles of spherical size and an average diameter of $40 \mathrm{~nm}$ in a vertical tube. These results show that the condensation heat transfer coefficient has been improved with the increase in nanoparticle concentration and steam mass flow. In addition, an increase in the degree of steam superheat leads to an increase in the thermal transfer coefficient.

1.4. Present Work. Although recent publications have shown positive impact of nanoparticles on heat transfer, including natural, mixed, or forced convection, for condensation, there are few studies on the impact of nanoparticles on this phase change phenomenon. In this article, the effect of additional nanoparticles in vapor phase on condensation of vapor in the presence of NCG (air) was studied for different volume fractions of nanoparticles, velocities, and relative humidities at the inlet of the vertical channel. The local values of the Nusselt number and the condensate thickness as well as the accumulated rate of condensation were discussed.

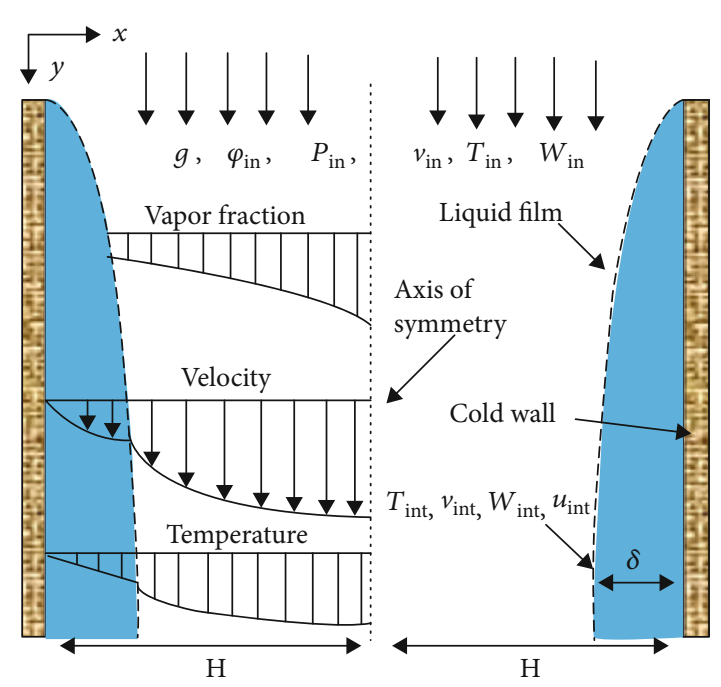

FIgURE 1: Scheme of the physical model under study.

\section{Physical Model and Mathematical Formulation}

We consider a water vapor with nanoparticles in presence of a NCG circulating in a symmetrical vertical channel of length $L$ and width of $2 H$ (Figure 1). Walls of channel are subjected to a constant temperature (isothermal). At the entrance of the channel arrives a mixture of gas and vapor with nanoparticles at a uniform temperature, velocity, pressure, and mass fraction of water vapor.

2.1. Simplifying Assumptions of the Problem. Simplifying assumptions were adopted for both phases. These are listed below:

(i) Gas flow is laminar and stationary

(ii) Heat and mass transfers are supposed to be two dimensional

(iii) Vapor-air mixture is considered an ideal mixture

(iv) Radiation heat transfer, the viscous dissipation, and the pressure work terms are neglected in the energy equation

(v) There is a local thermal equilibrium between nanoparticles and main flow

(vi) Nanoparticles are distributed evenly in air-vapor mixture and in the liquid film

(vii) Liquid film contains the same fraction of the nanoparticles as in the mixture.

2.2. Liquid Film with Nanoparticles Model. Equations used to describe heat and mass transfer phenomenon during condensation are given in the following references $[13,19,28$, 
29]. According to assumptions mentioned above, liquid phase equations are written as follows:

(i) Mass conservation:

$$
\frac{\partial}{\partial x}\left(\rho_{n f, L} u_{L}\right)+\frac{\partial}{\partial y}\left(\rho_{n f, L} v_{L}\right)=0
$$

(ii) Momentum conservation:

$$
\begin{gathered}
\frac{\partial}{\partial x}\left(\rho_{n f, L} u_{L} u_{L}\right)+\frac{\partial}{\partial y}\left(\rho_{n f, L} v_{L} u_{L}\right) \\
=-\frac{\partial P_{L}}{\partial x}+\frac{\partial}{\partial x}\left(\mu_{n f, L} \frac{\partial u_{L}}{\partial x}\right)+\frac{\partial}{\partial y}\left(\mu_{n f, L} \frac{\partial u_{L}}{\partial y}\right), \\
\frac{\partial}{\partial x}\left(\rho_{n f, L} u_{L} v_{L}\right)+\frac{\partial}{\partial y}\left(\rho_{n f, L} v_{n f, L} v_{n f, L}\right) \\
=-\frac{\partial P_{L}}{\partial y}+\frac{\partial}{\partial x}\left(\mu_{n f, L} \frac{\partial v_{L}}{\partial x}\right)+\frac{\partial}{\partial y}\left(\mu_{n f, L} \frac{\partial v_{L}}{\partial y}\right)+\rho_{n f, L} g
\end{gathered}
$$

(iii) Energy conservation:

$$
\begin{gathered}
\frac{\partial}{\partial x}\left(\rho_{n f, L} C_{P, n f, L} u_{L} T_{L}\right)+\frac{\partial}{\partial y}\left(\rho_{n f, L} C_{P, n f, L} v_{L} T_{L}\right) \\
=\frac{\partial}{\partial x}\left(\lambda_{n f, L} \frac{\partial T_{L}}{\partial x}\right)+\frac{\partial}{\partial y}\left(\lambda_{n f, L} \frac{\partial T_{L}}{\partial y}\right) .
\end{gathered}
$$

2.3. Humid Air-Nanoparticles Mixture Model. The conservation equations in the field of mixture have been formulated taking into consideration the simplifying assumptions mentioned above. Afterwards, the equations of the mixing range can be transcribed as follows:

(i) Mass conservation:

$$
\frac{\partial}{\partial x}\left(\rho_{n f, M} u_{M}\right)+\frac{\partial}{\partial y}\left(\rho_{n f, M} v_{M}\right)=0
$$

(ii) Momentum conservation:

$$
\begin{aligned}
& \frac{\partial}{\partial x}\left(\rho_{n f, M} u_{M} u_{M}\right)+\frac{\partial}{\partial y}\left(\rho_{n f, M} v_{M} u_{M}\right) \\
& \quad=-\frac{\partial P_{M}}{\partial x}+\frac{\partial}{\partial x}\left(\mu_{n f, M} \frac{\partial u_{M}}{\partial x}\right)+\frac{\partial}{\partial y}\left(\mu_{n f, M} \frac{\partial u_{M}}{\partial y}\right),
\end{aligned}
$$

$$
\begin{aligned}
& \frac{\partial}{\partial x}\left(\rho_{n f, M} u_{M} v_{M}\right)+\frac{\partial}{\partial y}\left(\rho_{n f, M} v_{M} v_{M}\right) \\
& \quad=-\frac{\partial P_{M}}{\partial y}+\frac{\partial}{\partial x}\left(\mu_{n f, M} \frac{\partial v_{M}}{\partial x}\right)+\frac{\partial}{\partial y}\left(\mu_{n f, M} \frac{\partial v_{M}}{\partial y}\right)+\rho_{n f, M} g
\end{aligned}
$$

(iii) Energy conservation:

$$
\begin{gathered}
\frac{\partial}{\partial x}\left(\rho_{n f, M} C_{P, n f, M} u_{M} T_{M}\right)+\frac{\partial}{\partial y}\left(\rho_{n f, M} C_{P, n f, M} v_{M} T_{M}\right) \\
=\frac{\partial}{\partial x}\left(\lambda_{n f, M} \frac{\partial T_{M}}{\partial x}\right)+\frac{\partial}{\partial y}\left(\lambda_{n f, M} \frac{\partial T_{M}}{\partial y}\right)
\end{gathered}
$$

(iv) Species conservation:

$$
\begin{aligned}
& \frac{\partial}{\partial x}\left(\rho_{n f, M} u_{M} W\right)+\frac{\partial}{\partial y}\left(\rho_{n f, M} v_{M} W\right) \\
& \quad=\frac{\partial}{\partial x}\left(\rho_{n f, M} D \frac{\partial W}{\partial x}\right)+\frac{\partial}{\partial y}\left(\rho_{n f, M} D \frac{\partial W}{\partial y}\right) .
\end{aligned}
$$

2.4. Coordinate Transformation for Both Phases. In order to remedy nonuniformity of mesh generated by the profile of the liquid film thickness, we have changed the variables from coordinates $(x, y)$ to coordinates $(\eta, \chi)$, such a change is essential to clarify interface location:

$y=\chi$ for $0 \leq y \leq L$

$\eta=x / \delta$ for $0 \leq x \leq \delta$

$\eta=(x-\delta) /(H-\delta)+1$ for $\delta \leq x \leq H$.

Following the aforementioned change of variables, the governing equations are thus transformed as follows:

(i) Continuity equation in the liquid film:

$$
\frac{1}{\delta} \frac{\partial\left(\rho_{n f, L} u_{L}\right)}{\partial \eta}+\frac{\partial\left(\rho_{n f, L} v_{L}\right)}{\partial \chi}=\frac{\eta}{\delta} \frac{\partial \delta}{\partial \chi} \frac{\partial\left(\rho_{n f, L} v_{L}\right)}{\partial \eta}
$$

(ii) Momentum equation in the liquid film: 


$$
\begin{gathered}
\frac{1}{\delta} \frac{\partial\left(\rho_{n f, L} u_{L} u_{L}\right)}{\partial \eta}+\frac{\partial\left(\rho_{n f, L} v_{L} u_{L}\right)}{\partial \chi} \\
=-\frac{1}{\delta} \frac{\partial P_{L}}{\partial \eta}+\frac{1}{\delta^{2}} \frac{\partial}{\partial \eta}\left(\mu_{n f, L} \frac{\partial u_{L}}{\partial \eta}\right)+\frac{\partial}{\partial \chi} \\
\cdot\left[\mu_{n f, L}\left(\frac{\partial u_{L}}{\partial \chi}-\frac{\eta}{\delta} \frac{\partial \delta}{\partial \chi} \frac{\partial u_{L}}{\partial \eta}\right)\right]-\frac{\eta}{\delta} \frac{\partial \delta}{\partial \chi} \frac{\partial}{\partial \eta} \\
\cdot\left[\mu_{n f, L}\left(\frac{\partial u_{L}}{\partial \chi}-\frac{\eta}{\delta} \frac{\partial \delta}{\partial \chi} \frac{\partial u_{L}}{\partial \eta}\right)\right]+\frac{\eta}{\delta} \frac{\partial \delta}{\partial \chi} \frac{\partial\left(\rho_{n f, L} v_{L} u_{L}\right)}{\partial \eta} \\
\frac{1}{\delta} \frac{\partial\left(\rho_{n f, L} u_{L} v_{L}\right)}{\partial \eta}+\frac{\partial\left(\rho_{n f, L} v_{L} v_{L}\right)}{\partial \chi} \\
=-\frac{\partial P_{L}}{\partial \chi}+\frac{\eta}{\delta} \frac{\partial \delta}{\partial \chi} \frac{\partial P_{L}}{\partial \eta}+\frac{1}{\delta^{2}} \frac{\partial}{\partial \eta}\left(\mu_{n f, L} \frac{\partial v_{L}}{\partial \eta}\right) \\
+\frac{\partial}{\partial \chi}\left[\mu_{n f, L}\left(\frac{\partial v_{L}}{\partial \chi}-\frac{\eta}{\delta} \frac{\partial \delta}{\partial \chi} \frac{\partial v_{L}}{\partial \eta}\right)\right]-\frac{\eta}{\delta} \frac{\partial \delta}{\partial \chi} \frac{\partial}{\partial \eta} \\
\quad \cdot\left[\mu_{n f, L}\left(\frac{\partial v_{L}}{\partial \chi}-\frac{\eta}{\delta} \frac{\partial \delta}{\partial \chi} \frac{\partial v_{L}}{\partial \eta}\right)\right] \\
+\frac{\eta}{\delta} \frac{\partial \delta}{\partial \chi} \frac{\partial\left(\rho_{n f, L} v_{L} v_{L}\right)}{\partial \eta}+g \rho_{n f, L}
\end{gathered}
$$

(iii) Energy equation in the liquid film:

$$
\begin{aligned}
\frac{1}{\delta} & \frac{\partial\left(\rho_{n f, L} C_{P, n f, L} u_{L} T_{L}\right)}{\partial \eta}+\frac{\partial\left(\rho_{n f, L} C_{P, n f, L} \nu_{L} T_{L}\right)}{\partial \chi} \\
= & \frac{1}{\delta^{2}} \frac{\partial}{\partial \eta}\left(\lambda_{n f, L} \frac{\partial T_{L}}{\partial \eta}\right)+\frac{\partial}{\partial \chi}\left[\lambda_{n f, L}\left(\frac{\partial T_{L}}{\partial \chi}-\frac{\eta}{\delta} \frac{\partial \delta}{\partial \chi} \frac{\partial T_{L}}{\partial \eta}\right)\right] \\
& -\frac{\eta}{\delta} \frac{\partial \delta}{\partial \chi} \frac{\partial}{\partial \eta}\left[\lambda_{n f, L}\left(\frac{\partial T_{L}}{\partial \chi}-\frac{\eta}{\delta} \frac{\partial \delta}{\partial \chi} \frac{\partial T_{L}}{\partial \eta}\right)\right] \\
& +\frac{\eta}{\delta} \frac{\partial \delta}{\partial \chi} \frac{\partial\left(\rho_{n f, L} C_{P, n f, L} \nu_{L} T_{L}\right)}{\partial \eta}
\end{aligned}
$$

(i) Continuity equation in the mixture region:

$$
\frac{1}{H-\delta} \frac{\partial\left(\rho_{n f, M} u_{M}\right)}{\partial \eta}+\frac{\partial\left(\rho_{n f, M} v_{M}\right)}{\partial \chi}=\frac{2-\eta}{H-\delta} \frac{\partial \delta}{\partial \chi} \frac{\partial\left(\rho_{n f, M} v_{M}\right)}{\partial \eta}
$$

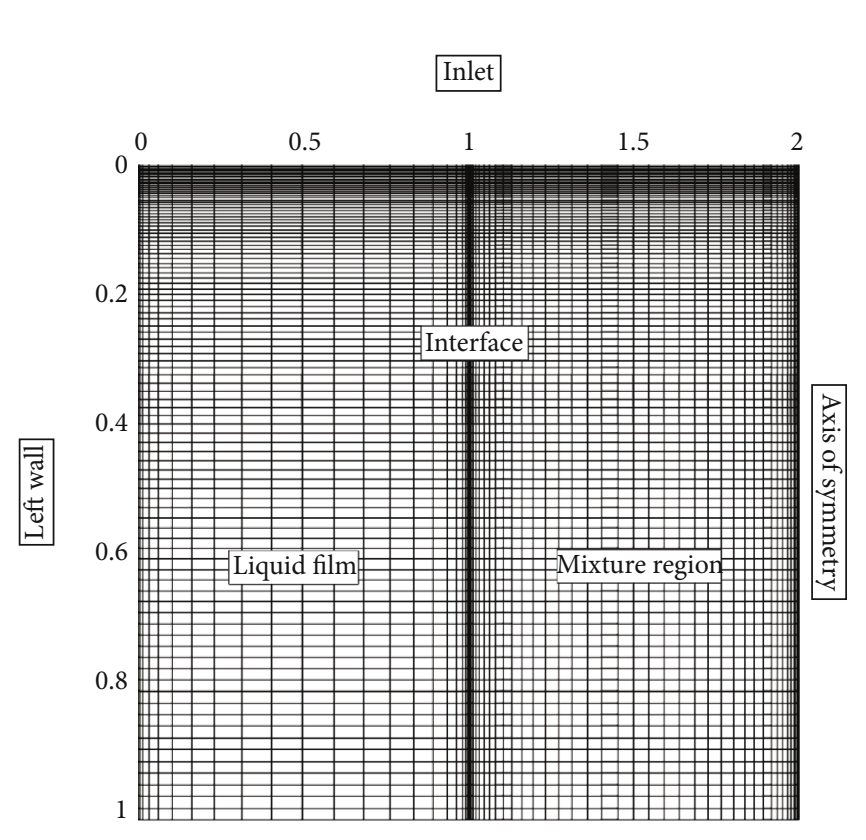

Figure 2: Mesh distribution in the numerical domain.

\begin{tabular}{lc}
\hline Density $\left(\mathrm{kg} \mathrm{m}^{-1}\right)$ & 8300 \\
Thermal conductivity $\left(\mathrm{J} \mathrm{s}^{-1} \mathrm{~K}^{-1}\right)$ & 401 \\
Heat capacity $\left(\mathrm{J} \mathrm{s}^{-1} \mathrm{~kg} \mathrm{~K}^{-1}\right)$ & 420 \\
\hline
\end{tabular}

$$
\begin{aligned}
\frac{1}{H-\delta} & \frac{\partial\left(\rho_{n f, M} u_{M} u_{M}\right)}{\partial \eta}+\frac{\partial\left(\rho_{n f, M} v_{M} u_{M}\right)}{\partial \chi} \\
= & -\frac{1}{H-\delta} \frac{\partial P_{M}}{\partial \eta}+\frac{1}{(H-\delta)^{2}} \frac{\partial}{\partial \eta}\left(\mu_{n f, M} \frac{\partial u_{M}}{\partial \eta}\right) \\
& +\frac{\partial}{\partial \chi}\left[\mu_{n f, M}\left(\frac{\partial u_{M}}{\partial \chi}-\frac{2-\eta}{H-\delta} \frac{\partial \delta}{\partial \chi} \frac{\partial u_{M}}{\partial \eta}\right)\right] \\
& -\frac{2-\eta}{H-\delta} \frac{\partial \delta}{\partial \chi} \frac{\partial}{\partial \eta}\left[\mu_{n f, M}\left(\frac{\partial u_{M}}{\partial \chi}-\frac{2-\eta}{H-\delta} \frac{\partial \delta}{\partial \chi} \frac{\partial u_{M}}{\partial \eta}\right)\right] \\
& +\frac{2-\eta}{H-\delta} \frac{\partial \delta}{\partial \chi} \frac{\partial\left(\rho_{n f, M} v_{M} u_{M}\right)}{\partial \eta}, \\
\frac{1}{H-\delta} & \frac{\partial\left(\rho_{n f, M} u_{M} v_{M}\right)}{\partial \eta}+\frac{\partial\left(\rho_{n f, M} v_{M} v_{M}\right)}{\partial \chi} \\
= & -\frac{\partial P_{M}}{\partial \chi}+\frac{2-\eta}{H-\delta} \frac{\partial \delta}{\partial \chi} \frac{\partial P_{M}}{\partial \eta}+\frac{1}{(H-\delta)^{2}} \frac{\partial}{\partial \eta}\left(\mu_{n f, M} \frac{\partial v_{M}}{\partial \eta}\right) \\
& +\frac{\partial}{\partial \chi}\left[\mu_{n f, M}\left(\frac{\partial v_{M}}{\partial \chi}-\frac{2-\eta}{H-\delta} \frac{\partial \delta}{\partial \chi} \frac{\partial v_{M}}{\partial \eta}\right)\right] \\
& -\frac{2-\eta}{H-\delta} \frac{\partial \delta}{\partial \chi} \frac{\partial}{\partial \eta}\left[\mu_{n f, M}\left(\frac{\partial v_{M}}{\partial \chi}-\frac{2-\eta}{H-\delta} \frac{\partial \delta}{\partial \chi} \frac{\partial v_{M}}{\partial \eta}\right)\right] \\
& +\frac{2-\eta}{H-\delta} \frac{\partial \delta}{\partial \chi} \frac{\partial\left(\rho_{n f, M} v_{M} v_{M}\right)}{\partial \eta}+g \rho_{n f, M}
\end{aligned}
$$

(iii) Energy equation in the mixture region: 
TABLE 2: Grid sensitivity tests.

\begin{tabular}{|c|c|c|c|c|c|c|c|c|c|c|}
\hline \multirow{2}{*}{$\begin{array}{l}\chi \\
0.01007\end{array}$} & \multicolumn{2}{|c|}{$\begin{array}{c}(41 \times 51 \times 301) / \\
\left(\mathrm{Nu}_{\chi} \mathrm{Sh}_{\chi}\right)\end{array}$} & \multicolumn{2}{|c|}{$\begin{array}{c}(41 \times 71 \times 501) / \\
\left(\mathrm{Nu}_{\chi} \mathrm{Sh}_{\chi}\right)\end{array}$} & \multicolumn{2}{|c|}{$\begin{array}{c}(31 \times 51 \times 701) / \\
\left(\mathrm{Nu}_{\chi} \mathrm{Sh}_{\chi}\right)\end{array}$} & \multicolumn{2}{|c|}{$\begin{array}{c}(21 \times 51 \times 901) / \\
\left(\mathrm{Nu}_{\chi} \mathrm{Sh}_{\chi}\right)\end{array}$} & \multicolumn{2}{|c|}{$\begin{array}{c}(101 \times 101 \times 1001) \\
\left(\mathrm{Nu}_{\chi} \mathrm{Sh}_{\chi}\right)\end{array}$} \\
\hline & 85.534 & 15.108 & 95.118 & 16.738 & 97.866 & 17.204 & 98.601 & 17.326 & 98.440 & 17.291 \\
\hline 0.10079 & 37.259 & 6.619 & 37.614 & 6.681 & 37.713 & 6.700 & 37.697 & 6.697 & 37.563 & 6.6712 \\
\hline 0.20044 & 30.155 & 5.399 & 30.261 & 5.419 & 30.275 & 5.423 & 30.279 & 5.424 & 30.132 & 5.3960 \\
\hline 0.39945 & 25.487 & 4.627 & 25.465 & 4.625 & 25.520 & 4.637 & 25.526 & 4.638 & 25.378 & 4.6099 \\
\hline 0.75015 & 23.019 & 4.276 & 22.992 & 4.274 & 23.065 & 4.288 & 23.062 & 4.288 & 22.927 & 4.2622 \\
\hline 0.99367 & 22.255 & 4.197 & 22.245 & 4.198 & 22.310 & 4.212 & 22.307 & 4.211 & 22.178 & 4.1861 \\
\hline Err. Max. & $13.11 \%$ & $12.62 \%$ & $3.37 \%$ & $3.19 \%$ & $0.60 \%$ & $0.62 \%$ & $0.59 \%$ & $0.614 \%$ & Ref. & Ref. \\
\hline
\end{tabular}

$$
\begin{aligned}
\frac{1}{H-}-\delta & \frac{\partial\left(\rho_{n f, M} C_{P, n f, M} u_{M} T_{M}\right)}{\partial \eta}+\frac{\partial\left(\rho_{n f, M} C_{P, n f, M} v_{M} T_{M}\right)}{\partial \chi} \\
= & \frac{1}{(H-\delta)^{2}} \frac{\partial}{\partial \eta}\left(\lambda_{n f, M} \frac{\partial T_{M}}{\partial \eta}\right)+\frac{\partial}{\partial \chi} \\
& \cdot\left[\lambda_{n f, M}\left(\frac{\partial T_{M}}{\partial \chi}-\frac{2-\eta}{H-\delta} \frac{\partial \delta}{\partial \chi} \frac{\partial T_{M}}{\partial \eta}\right)\right]-\frac{2-\eta}{H-\delta} \frac{\partial \delta}{\partial \chi} \frac{\partial}{\partial \eta} \\
& \cdot\left[\lambda_{n f, M}\left(\frac{\partial T_{M}}{\partial \chi}-\frac{2-\eta}{H-\delta} \frac{\partial \delta}{\partial \chi} \frac{\partial T_{M}}{\partial \eta}\right)\right] \\
+ & \frac{2-\eta}{H-\delta} \frac{\partial \delta}{\partial \chi} \frac{\partial\left(\rho_{n f, M} C_{P, n f, M} v_{M} T_{M}\right)}{\partial \eta}
\end{aligned}
$$

(iv) Diffusion equation in the mixture region:

$$
\begin{aligned}
\frac{1}{H-}-\delta & \frac{\partial\left(\rho_{n f, M} u_{M} W\right)}{\partial \eta}+\frac{\partial\left(\rho_{n f, M} v_{M} W\right)}{\partial \chi} \\
= & \frac{1}{(H-\delta)^{2}} \frac{\partial}{\partial \eta}\left(\rho_{n f, M} D \frac{\partial W}{\partial \eta}\right)+\frac{\partial}{\partial \chi} \\
& \cdot\left[\rho_{n f, M} D\left(\frac{\partial W}{\partial \chi}-\frac{2-\eta}{H-\delta} \frac{\partial \delta}{\partial \chi} \frac{\partial W}{\partial \eta}\right)\right]-\frac{2-\eta}{H-\delta} \frac{\partial \delta}{\partial \chi} \frac{\partial}{\partial \eta} \\
& \cdot\left[\rho_{n f, M} D\left(\frac{\partial W}{\partial \chi}-\frac{2-\eta}{H-\delta} \frac{\partial \delta}{\partial \chi} \frac{\partial W}{\partial \eta}\right)\right] \\
& +\frac{2-\eta}{H-\delta} \frac{\partial \delta}{\partial \chi} \frac{\partial\left(\rho_{n f, M} v_{M} W\right)}{\partial \eta} .
\end{aligned}
$$

2.5. Boundary Conditions. The boundary conditions in the transformed coordinates are prescribed below $[13,19,28$, 29]:

(i) At the entrance of the canal $\chi=0$ :

$$
v_{L}=u_{L}=u_{M}=0 . \quad ; \quad v_{M}=v_{\text {in }} ; T_{L}=T_{W} ; T_{M}=T_{\text {in }} ; W=W_{\text {in }}
$$

(ii) At the outlet of the canal $\chi=L$ :

$\frac{\partial u_{L}}{\partial \chi}=\frac{\partial v_{L}}{\partial \chi}=\frac{\partial T_{L}}{\partial \chi}=\frac{\partial u_{M}}{\partial \chi}=\frac{\partial v_{M}}{\partial \chi}=\frac{\partial T_{M}}{\partial \chi}=\frac{\partial W}{\partial \chi}=0$

(iii) On the left wall $\eta=0$ :

$$
u_{L}=0 ; v_{L}=0 ; T_{L}=T_{W}
$$

(iv) At the interface $\eta=1$ :

$$
\begin{gathered}
\left.v_{L}\right|_{\text {int }}=\left.v_{M}\right|_{\text {int }}, \\
\left.\frac{\mu_{L}}{\delta} \frac{\partial v_{L}}{\partial \eta}\right|_{\text {int }}=\left.\frac{\mu_{M}}{H-\delta} \frac{\partial v_{M}}{\partial \eta}\right|_{\text {int }}, \\
\left.T_{L}\right|_{\text {int }}=\left.T_{M}\right|_{\text {int }}=T_{\text {sat }}, \\
J_{\text {int }}^{\prime \prime}=-\left.\frac{\rho_{n f, M} D}{\left(1-W_{\text {int }}\right)(H-\delta)} \frac{\partial W}{\partial \eta}\right|_{\text {int }}, \\
\left.\frac{\lambda_{n f, L}}{\delta} \frac{\partial T_{L}}{\partial \eta}\right|_{\text {int }}=\left.\frac{\lambda_{n f, M}}{H-\delta} \frac{\partial T_{M}}{\partial \eta}\right|_{\text {int }}-J_{\text {int }}^{\prime \prime} h_{f g}, \\
\rho_{n f, L}\left(u_{L}-v_{L} \frac{d \delta}{d \chi}\right)_{\text {int }}=\rho_{n f, M}\left(u_{M}-v_{M} \frac{d \delta}{d \chi}\right)_{\text {int }}=J_{\text {int }}^{\prime \prime}
\end{gathered}
$$

(v) On the axis of symmetry $\eta=2$ : 


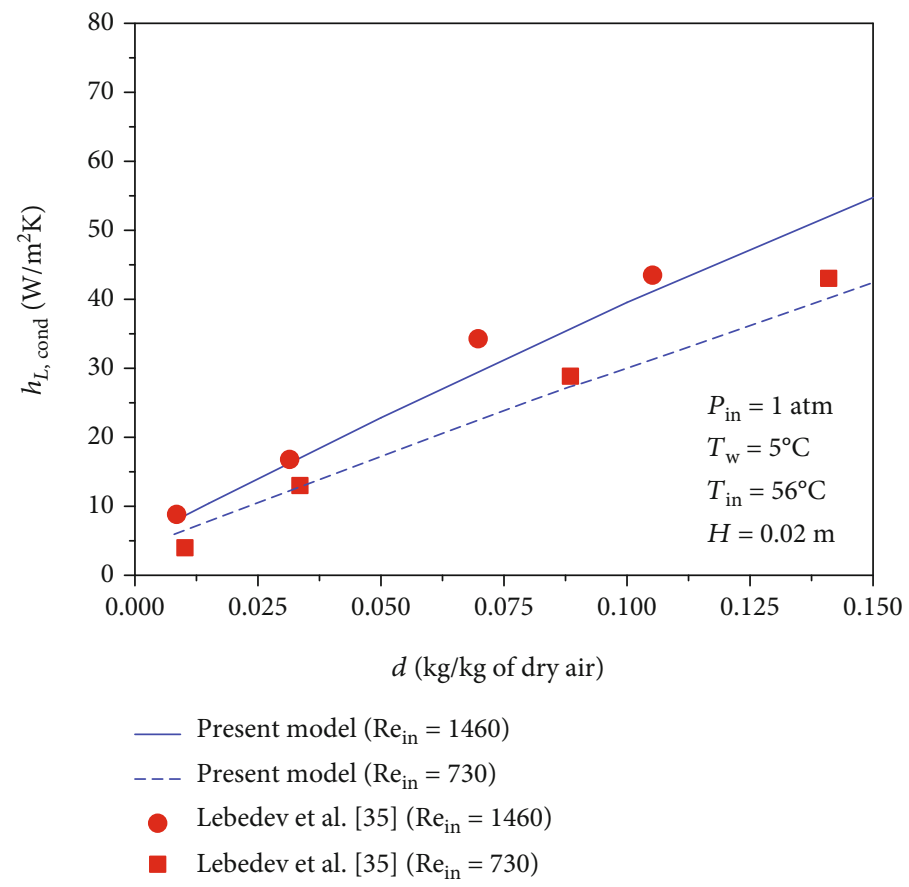

FIGURE 3: Validation with Lebedev et al. [35].

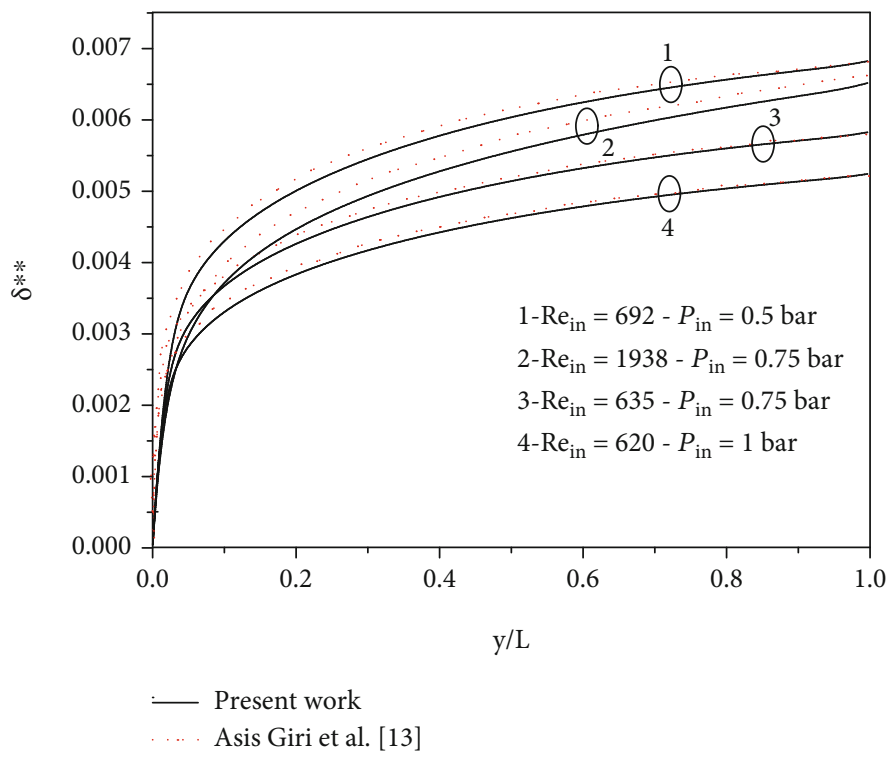

Figure 4: Validation with Giri et al. [13].

TABLE 3: Validation of the solution with experimental data of [35].

\begin{tabular}{lcccccc}
\hline & \multicolumn{3}{c}{$\mathrm{Re}_{\mathrm{in}}=730$} & \multicolumn{3}{c}{$\mathrm{Re}_{\mathrm{in}}=1460$} \\
& $\begin{array}{c}\text { Present } \\
\text { work }\end{array}$ & {$[35]$} & $\begin{array}{c}\text { Relative } \\
\text { error }\end{array}$ & $\begin{array}{c}\text { Present } \\
\text { work }\end{array}$ & {$[35]$} & $\begin{array}{c}\text { Relative } \\
\text { error }\end{array}$ \\
\hline 0.05 & 17.381 & 15.40 & $12 \%$ & 22.8398 & 23.46 & $2.6 \%$ \\
0.1 & 30.2757 & 31.46 & $3.7 \%$ & 39.5541 & 45.38 & $12 \%$ \\
0.15 & 42.4438 & 48.4842 & $12 \%$ & 54.7401 & 68.12723 & $19 \%$ \\
\hline
\end{tabular}

$$
\mathrm{u}_{M}=0 \quad ; \quad \frac{\partial v_{M}}{\partial \eta}=\frac{\partial T_{M}}{\partial \eta}=\frac{\partial W}{\partial \eta}=0
$$

2.6. Nanoparticles Proprieties. The properties of nanofluids (condensate with nanoparticles and air-vapor-nanoparticles) are calculated as a function of the volume fraction; the properties of solid nanoparticles listed in Table 1, and the base 
TABLE 4: Validation of the solution with numerical data of [13].

\begin{tabular}{lccccccccc}
\hline$\chi$ & & $\mathrm{Re}_{\mathrm{in}}=692$ & \multicolumn{3}{c}{$\mathrm{Re}_{\mathrm{in}}=1968$} & & \multicolumn{2}{c}{$\mathrm{Re}_{\text {in }}=620$} \\
{$[13]$} & Relative error \\
\hline 0.05 & 0.00361 & 0.00388 & $6.9 \%$ & 0.00298 & 0.00329 & $9.4 \%$ & 0.00282 & 0.00295 & $4.4 \%$ \\
0.22 & 0.00515 & 0.00529 & $2.6 \%$ & 0.00462 & 0.00487 & $5.1 \%$ & 0.00394 & 0.00401 & $1.7 \%$ \\
0.53 & 0.00611 & 0.00622 & $1.7 \%$ & 0.00564 & 0.00593 & $4.8 \%$ & 0.00468 & 0.00471 & $0.6 \%$ \\
1 & 0.00682 & 0.00681 & $0.11 \%$ & 0.00651 & 0.00661 & $1.5 \%$ & 0.00524 & 0.00521 & $0.5 \%$ \\
\hline
\end{tabular}

fluid. Thus, the properties of the nanofluid are given by the following relationships [30-33]:

$$
\begin{gathered}
\mu_{n f}=(1-\varphi)^{-2.5} \mu_{f}, \\
\rho_{n f}=(1-\varphi) \rho_{f}+\varphi \rho_{p}, \\
C_{P, n f}=(1-\varphi) C_{P, f}+\varphi C_{P, p}, \\
\lambda_{n f}=\lambda_{f} \frac{\lambda_{p}+2 \lambda_{f}-2 \varphi\left(\lambda_{f}-\lambda_{p}\right)}{\lambda_{p}+2 \lambda_{f}+2 \varphi\left(\lambda_{f}-\lambda_{p}\right)} .
\end{gathered}
$$

The thermophysical properties of $\mathrm{Cu}$ nanoparticles are listed in Table 1 [1].

2.7. Characteristic Variables. The local Nusselt number at interface is determined by the following expression:

$$
N u_{y}=\left.\frac{(2 H) \lambda_{n f, M}}{\lambda_{f}(H-\delta)\left(T_{\text {bulk }}-T_{\text {int }}\right)} \frac{\partial T_{M}}{\partial \eta}\right|_{\text {int }}-\frac{J_{\text {int }}^{\prime \prime} h_{f g}(2 H)}{\lambda_{f}\left(T_{\text {bulk }}-T_{\text {int }}\right)} .
$$

The bulk temperature is $T_{\text {bulk }}=\int_{\delta}^{H} \rho_{n f, M} C_{P, n f, M} v_{M} T_{M} d x$ $/ \int_{\delta}^{H} \rho_{n f, M} C_{P, n f, M} v_{M} d x$.

The accumulated condensation rate at interface is calculated by

$$
M r=-\int_{0}^{y} J_{\text {int }}^{\prime \prime} d y \text {. }
$$

The effect of the nanoparticles on the heat and mass transfers is determined by the effective ratio defined as follows:

$$
R_{\text {eff }}=\frac{\text { Variable in the case of nanofluid }}{\text { Variable in the case of base fluid }} .
$$

\section{Numerical Resolution Methods}

3.1. Computational Grid and Discretization. Based on change in variables mentioned above, the physical domain (Figure 1) can be transformed into two identical rectangular domains (Figure 2). Then, the variable position of the interface on $(x$

\begin{tabular}{|c|c|}
\hline Parameters & Ranges \\
\hline Nanoparticles volume fraction, $\varphi_{\text {in }}$ & $0.01 \%, 0.05 \%, 0.1 \%$ \\
\hline Reynolds number, $\mathrm{Re}_{\text {in }}$ & $1000,1500,2000$ \\
\hline Inlet relative humidity, $\mathrm{RH}_{\text {in }}$ & $50 \%, 75 \%, \quad 100 \%$ \\
\hline Inlet to wall temperature difference, $\Delta T$ & $20^{\circ} \mathrm{C}$ \\
\hline Inlet temperature, $T_{\text {in }}$ & $30^{\circ} \mathrm{C}, 40^{\circ} \mathrm{C}, 50^{\circ} \mathrm{C}$ \\
\hline Inlet pressure, $P_{\text {in }}$ & $0.5 \mathrm{~atm}, 1 \mathrm{~atm}, 2 \mathrm{~atm}$ \\
\hline
\end{tabular}
$, y)$ coordinates becomes a parallel line to wall in $(\chi, \eta)$ coordinates. A nonuniform computation grid with rectangular control volumes was used in both horizontal and vertical directions, as shown in Figure 2, using suitable functions, in order to obtain an appropriate refinement in areas with
TABLE 5: Operating conditions.

strong gradients: near the wall, near the entrance, and near the interface as shown in Figure 2.

The study of the influence of different grid on local Nusselt number variation at the interface and the local Sherwood number variation at the interface at various $\chi$ locations was conducted to fix the optimal mesh (see Table 2). Inlet conditions used for these tests are $\operatorname{Re}_{\text {in }}=1000, P_{\text {in }}=1$ atm, $T_{\text {in }}=$ $40^{\circ} \mathrm{C}, \mathrm{RH}_{\text {in }}=100 \%, \varphi_{\text {in }}=0 \%$, and $\Delta T=20^{\circ} \mathrm{C}$. Table 2 shows that, in the case of $\mathrm{NM}=51, \mathrm{NL}=21$, and $\mathrm{NY}=901$, the relative error for the local Nusselt number and condensed mass flow rate does not exceed $0.6 \%$ and $0.7 \%$, respectively, when compared with finer grids. These differences are considered suitably small, so that grid was used for all results presented in this work.

3.2. Simulation Proceeding. Discretization of equations (10)-(18) is performed using the finite volume method approach [34]. The SIMPLE algorithm (Semi-Implicit Method for Pressure-Linked Equations) is used for the velocity-pressure coupling. The power law scheme [34] is employed to handle convective and diffusion terms. The sweeping method line by line, with the algorithm of Thomas, was used for the iterative resolution of the systems of equations.

3.3. Condensate Film Thickness. Evolution of thickness of liquid film is determined by an iterative procedure using the Newton-Raphson method applied to the equation of the energy balance at the liquid-gas mixture interface.

$$
\frac{\lambda_{n f, L}}{\delta}\left(\frac{\partial T_{L}}{\partial \eta}\right)_{\text {int }}=\frac{\lambda_{n f, M}}{H-\delta}\left(\frac{\partial T_{M}}{\partial \eta}\right)_{\text {int }}+h_{f g} \frac{d}{d \chi} \int_{0}^{1} \rho_{n f, L} u_{L} \delta d \eta
$$




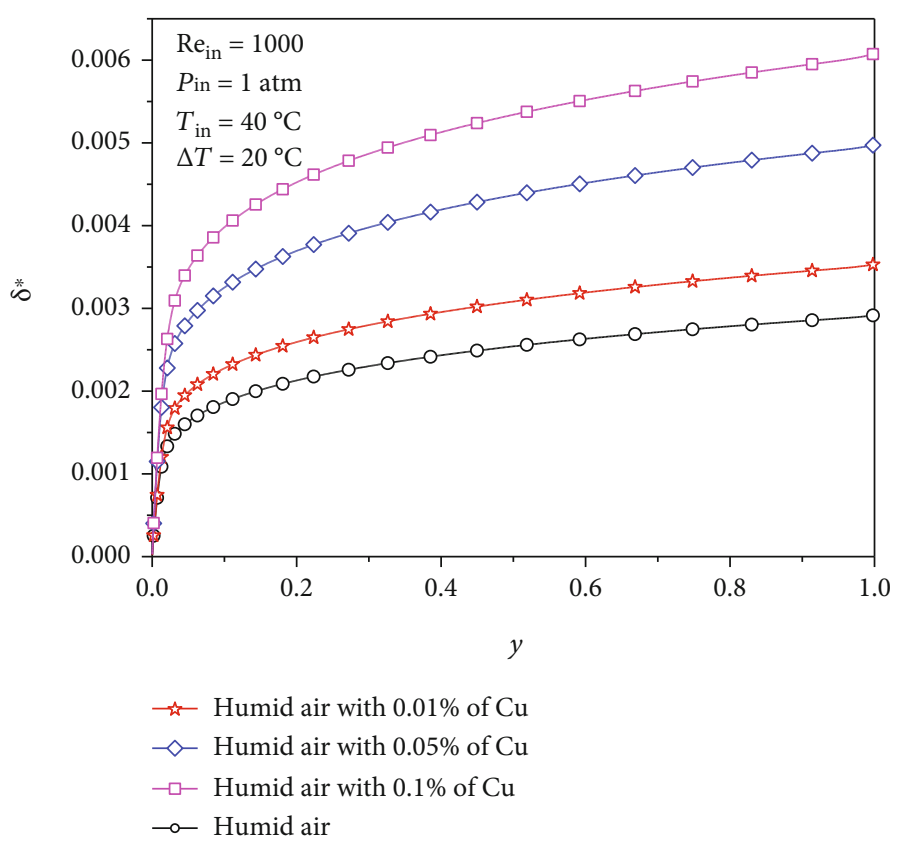

Figure 5: Condensate film thickness for different volume fractions of nanoparticles.

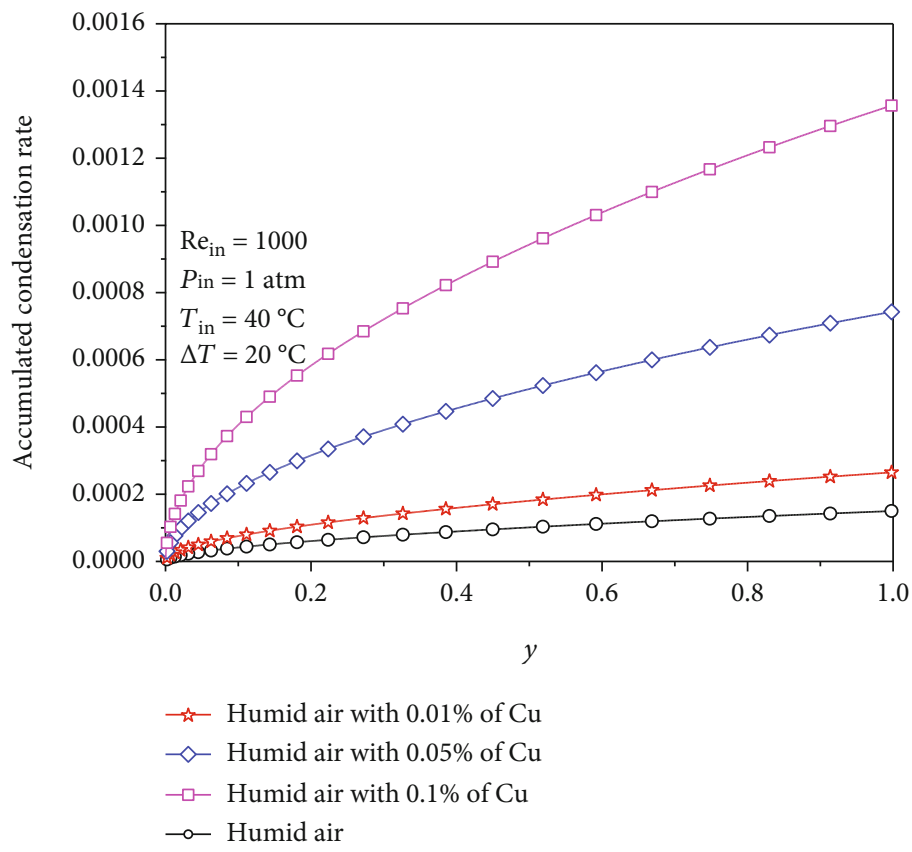

Figure 6: Accumulated condensation rate for different volume fractions of nanoparticles.

\section{Comparisons with Previous Works}

Validation tests are performed by comparison of our results with the experimental results of Lebedev et al. [35] and the numerical results of Giri et al. [13] (Figures 3 and 4 and Tables 3 and 4). Lebedev et al. [35] studied experimentally the heat transfer during condensation in an asymmetrically cooled channel of width $H=0.02 \mathrm{~m}$, the inlet numbers of Reynolds equal to $\operatorname{Re}_{\text {in }}=730$ and $\operatorname{Re}_{\text {in }}=1460$. Figure 3 and Table 4 show the comparison between the heat transfer coef- ficient as a function of the specific humidity found by the present model and that found by [35]. Regarding the numerical study, Giri et al. [13] studied the mixed convection heat transfer with condensation in a vertical channel, with the following data: $L=0.75 \mathrm{~m}, H=0.005 \mathrm{~m}, T_{\text {in }}=60^{\circ} \mathrm{C}, T_{\mathrm{W}}=30^{\circ}$ $\mathrm{C}$, and $\mathrm{RH}_{\text {in }}=100 \%$. Figure 4 and Table 4 show the comparison between our numerical results and those of [13]. A very satisfactory agreement was obtained between the results of the present model and those of the literature [35] and [13] (Tables 3 and 4). The small differences perceived between 


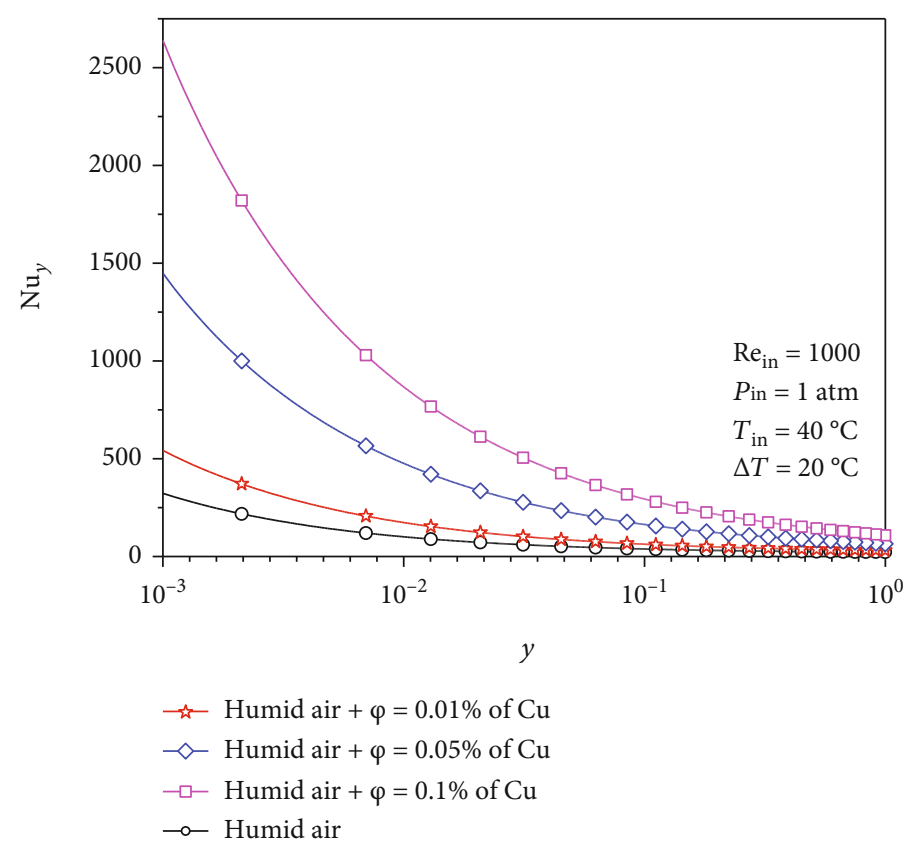

FIGURE 7: The local Nusselt number for different volume fractions of nanoparticles.

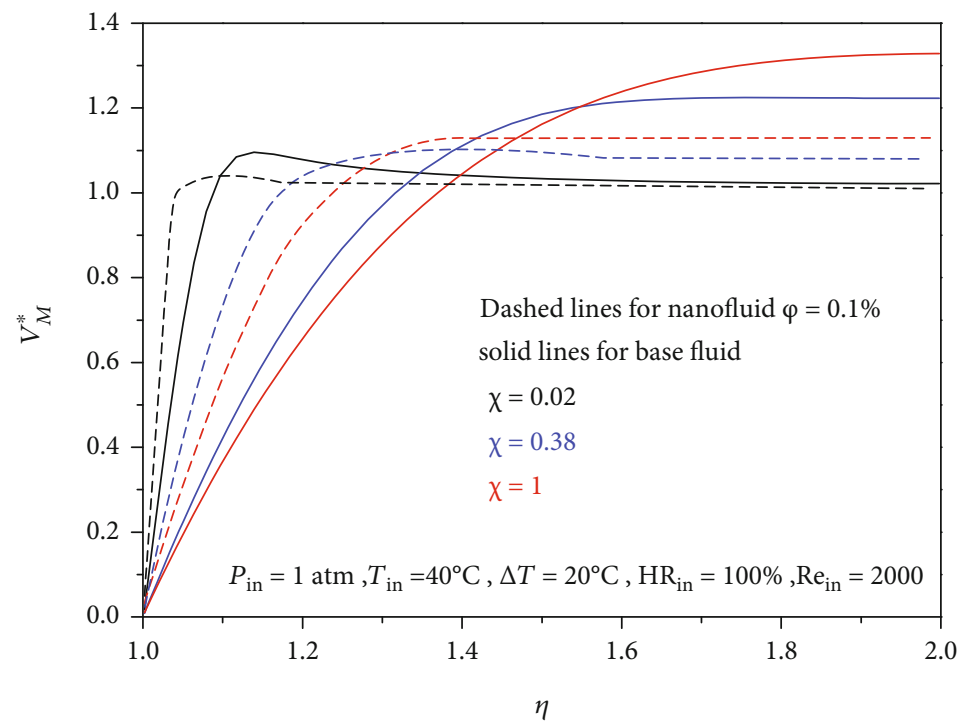

FIGURE 8: Dimensionless velocity profiles in the mixture region at different sections of the channel.

our results and those of Giri et al. [13] can be attributed to mathematical models used.

\section{Results and Discussion}

In this article, study of condensation of an air-vapor mixture in the presence of nanoparticles was carried out. The mixture flows downward into a condenser formed by vertical channel with length $L=1 \mathrm{~m}$ and half width $H=0.0125 \mathrm{~m}$. The walls of the channel are maintained isothermal. Calculations were made for the following data listed in Table 5.

5.1. Effects of Nanoparticles Volume Fraction. In order to evaluate the influence of the volume fraction of nanoparti- cles, Figures 5-7 show, respectively, the evolution of liquid film thickness, Nusselt number, and accumulated condensation rate for three volume fractions $0.01 \%, 0.05 \%$, and $0.1 \%$ of the $\mathrm{Cu}$ nanoparticles compared to the case of pure fluid.

Figure 5 illustrates the influence of variation in mass fraction of nanoparticles from $0 \%$ to $0.1 \%$ on the heat and mass transfer by condensation of nanofluid at an input Reynolds $\mathrm{Re}_{\text {in }}=1000$ and at $100 \%$ of relative humidity. From this figure, we can observe that the condensate film layer is very thin and that the slope of the film thickness evolution near the channel inlet region is high since the large concentration gradient occurs in this section. Moreover, moving away from the entrance, the evolution of the liquid film increasingly has moderate and substantially linear slopes for each fraction of 


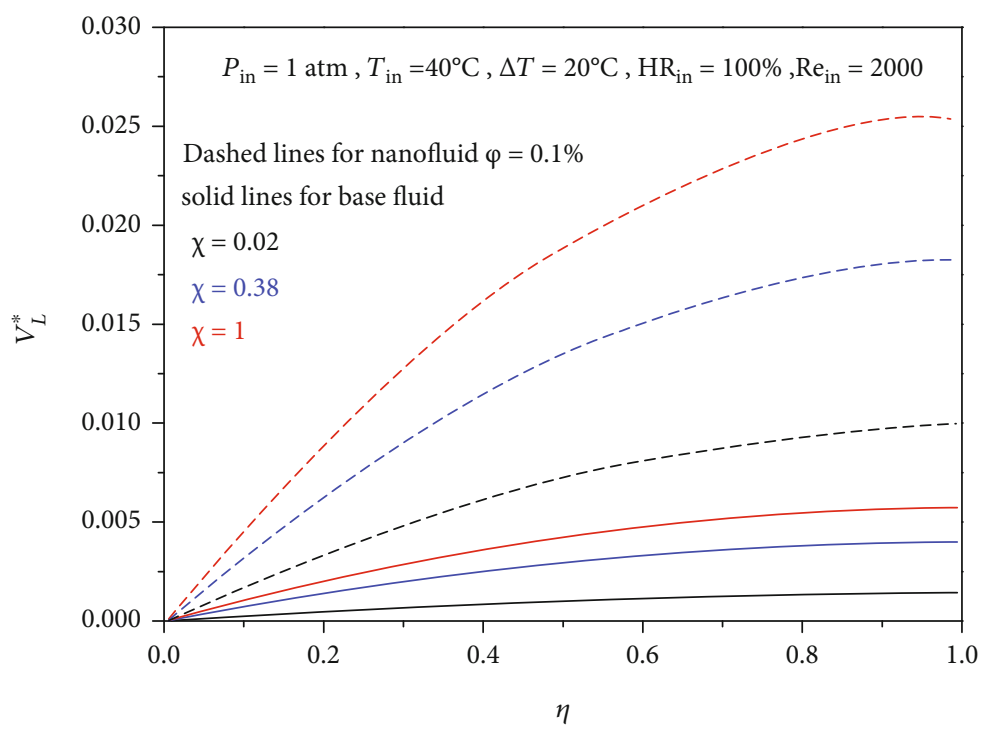

Figure 9: Dimensionless velocity profiles in the liquid region at different sections of the channel.

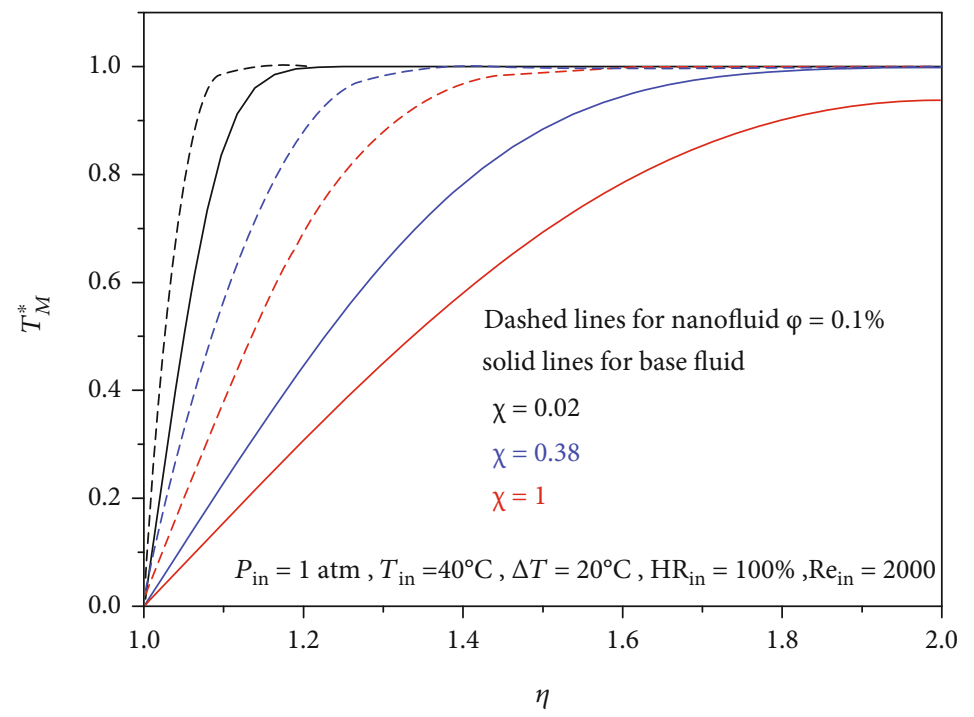

Figure 10: Dimensionless temperature profiles in the mixture region at different sections of the channel.

nanoparticles tested. Indeed, the amount of steam is high at the channel entrance and afterwards decreases due to the condensation process along the channel which leads to more important film thicknesses. This figure also indicates that as the volume fraction of the $\mathrm{Cu}$ nanoparticles increases from $0 \%$ to $0.1 \%$, the liquid film thickness improves by about $R_{\text {eff }}$ $=2$ at the outlet of the channel, indicating that more vapor is condensed in the presence of the nanoparticles. This trend is due to the improvement of the thermophysical properties of the base fluid. In particular, the density of the mixture is improved with the addition of the nanoparticles. The influence of the density is very important because its values are taken into account in the mass transfers through the diffusion coefficient.

The influence of the volume fraction of nanoparticles on the mass transfer is described by the variation in the conden- sation rate accumulated along the channel (Figure 6). This figure shows that increasing the volume fraction of the nanoparticles increases the mass flow rate of the condensate across the liquid-vapor interface. Therefore, the accumulated condensation flow rate increases significantly by increasing the volume fraction of the nanoparticles from $0 \%$ to $0.1 \%$. The maximum improvement of the accumulated condensation flow rate is about $R_{\text {eff }}=9.12$ at the outlet of the channel. The use of $\mathrm{Cu}$ nanoparticles leads to a better mass transfer as confirmed in Figure 5. It should be noted that the volume fractions of nanoparticle in nanofluid are very low (less than $0.1 \%)$ to be easily mixed with moist air, which is very useful to improve the convective transfer within the channel.

Figure 7 shows the evolution of the local Nusselt number at the interface for different volume fractions of nanoparticles. This figure shows that increasing the volume fraction 


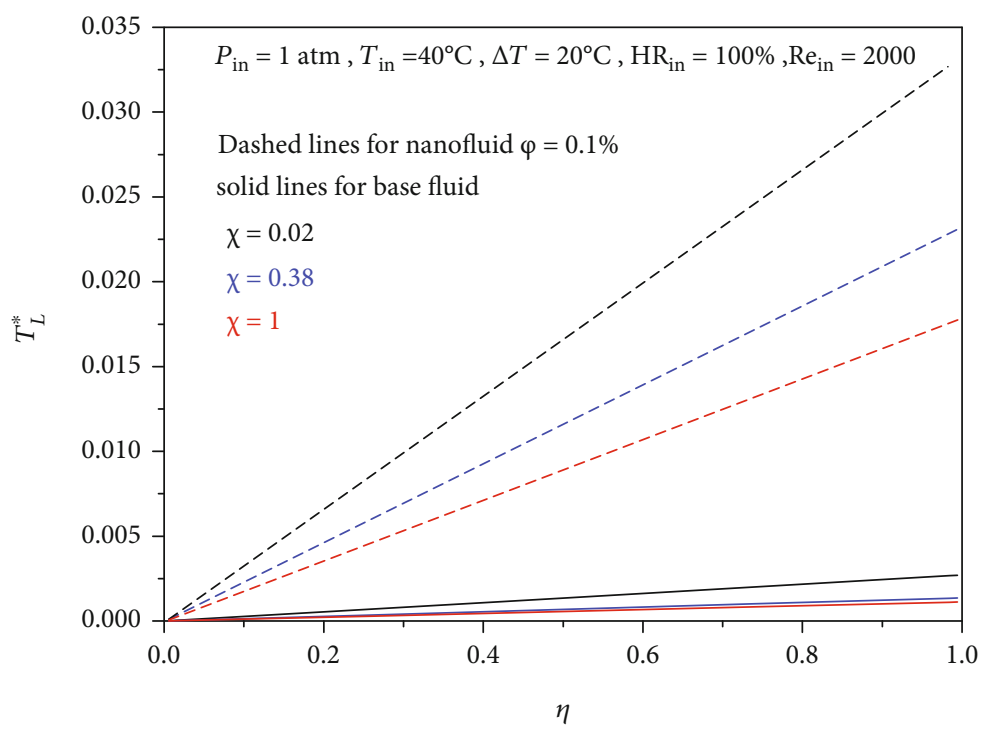

FIGURE 11: Dimensionless temperature profiles in the liquid region at different sections of the channel.

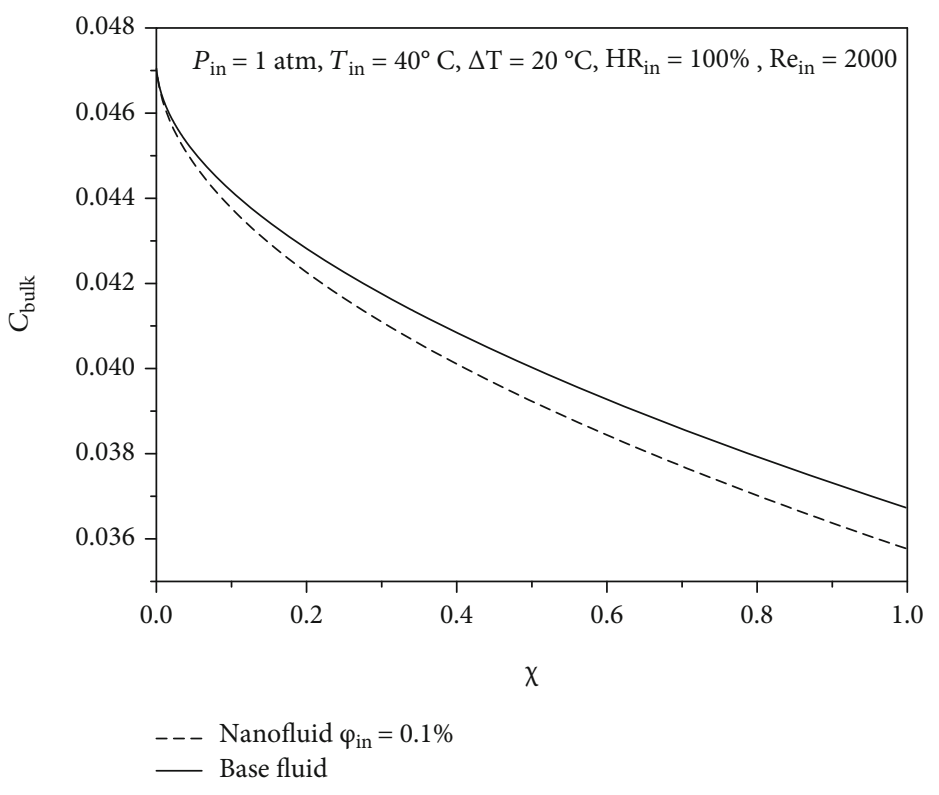

FiguRE 12: Bulk concentration profiles.

increases the local Nusselt number at the interface. Indeed, mass transfer is strongly coupled to heat transfer, so that a high mass transfer (Figures 5 and 6) leads to a high heat transfer from the interface to the liquid film. This figure also shows that for all volume fractions tested, the Nusselt number decreases rapidly away from the entrance area. This is due to the large temperature and vapor concentration gradients near the inlet; these gradients decrease axially along the channel. This decrease is due to the loss of steam through the condensation process. The heat transfer results mainly from the latent heat released by the condensation of steam, as the sensible heat due to thermal gradients in the gas mixture is relatively low. For a volume fraction of nanoparticles $\varphi=$ $0.1 \%$, the local Nusselt number improves by about $R_{\text {eff }}=$ 4.75 at the outlet of the channel.
5.2. Velocity, Temperature, and Bulk Concentration Profiles. The evolution of dimensionless velocity and temperature profiles in the liquid and gas phase regions at different axial cross sections of the channel is presented and analyzed in this section (Figures 8-12). The liquid film region corresponds to $0 \leq \eta \leq 1$, and the mixing region corresponds to $1 \leq \eta \leq 2$.

We can observe in Figures 8 and 9 in both cases with and without nanoparticles that when $\chi$ increases, the velocity of the air-vapor mixture tends to a parabolic profile, while the velocity of the liquid film increases along the channel but still remains low compared to the velocity of the gas phase. This trend is due to the low mass transfer from the vapor to the liquid across the interface. At different sections of the channel, we find that the velocity of the mixture decreases from the axis of symmetry of the channel to approach the velocity 


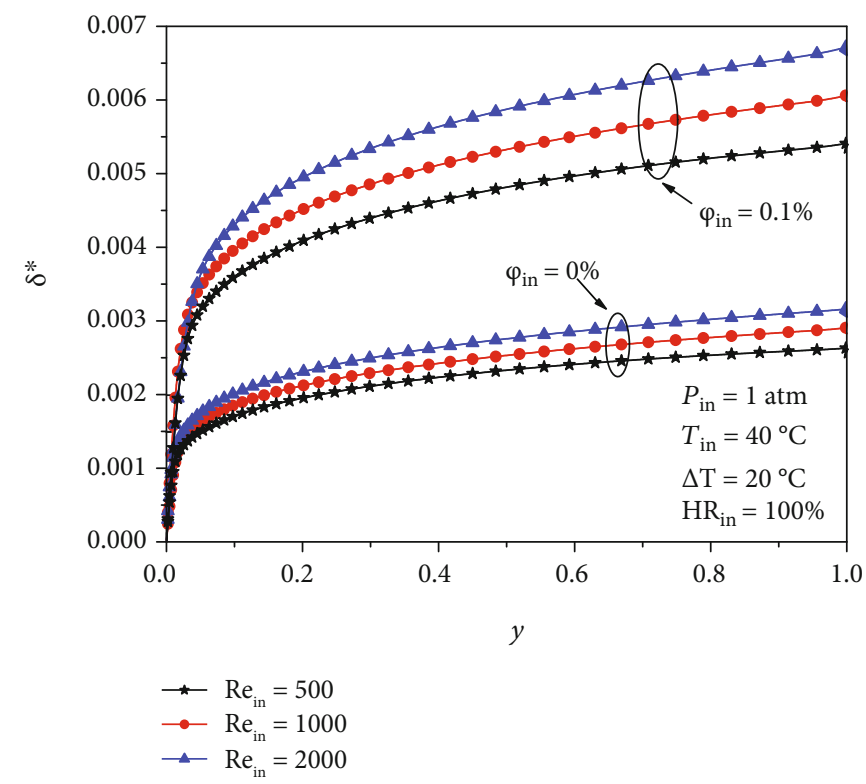

Figure 13: Effect of the inlet Reynolds number on the liquid film thickness.

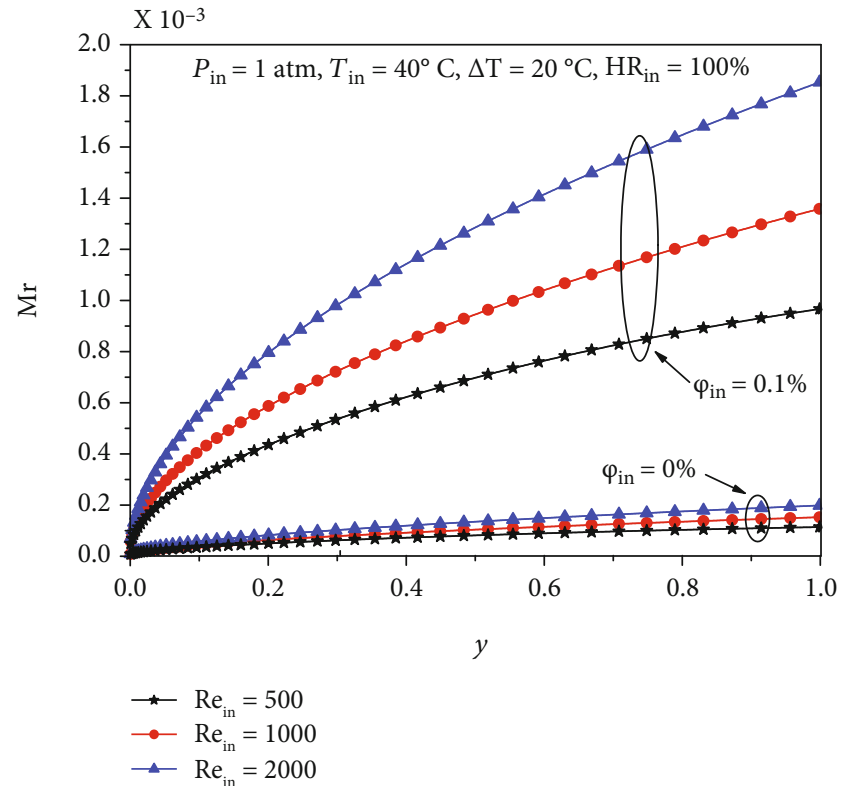

Figure 14: Effect of the inlet Reynolds number on accumulated condensation rate.

of the liquid phase at the liquid-vapor interface due to the effect of shear stress since the viscosity of the liquid is higher than that of the mixture at the liquid-vapor interface. In addition, the effect of the density and viscosity of the liquid leads to a very small increase in the velocity of the condensate (less than $3 \%$ of the inlet velocity) towards the channel outlet, as shown in Figure 9. The examination of the velocity profiles in the liquid region shows that the interfacial velocity increases along the channel, and all profiles are nearly parabolic. The vapor drives the liquid film, which moves slowly along the channel and resists the motion of the vapor as condensation continues.

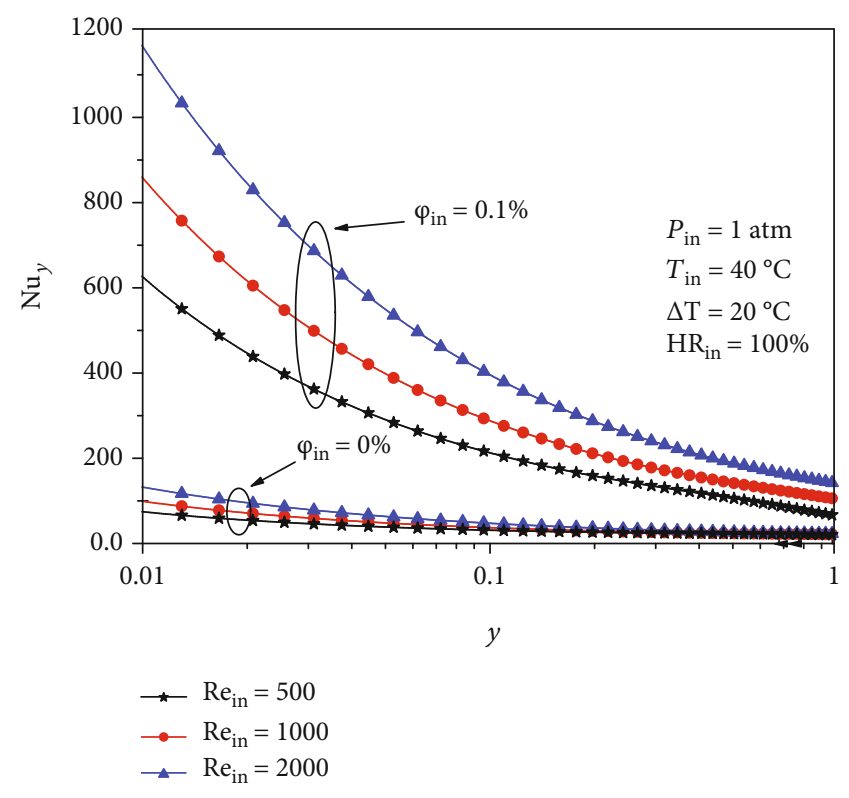

FIGURE 15: Effect of the input Reynolds number on the local Nusselt number.

Figures 10 and 11 show, respectively, the dimensionless temperature profiles in the two regions mixture and liquid at various axial cross sections with and without the nanoparticles. The temperature profiles in the liquid region are nearly linear, indicating that the effect of the convection terms in the energy equation is very small, and therefore, the transfer is nearly by conduction in this region. We also observe that the $T_{L}^{*}$ temperature at the interface is very low at all positions as shown in Figure 11. This is because the thickness of the liquid film is small, and therefore, the heat removal to the outside is very important. Figure 10 shows on the one hand that the temperature of the $T_{M}^{*}$ mixture starts at $T_{\text {in }}$ at the 


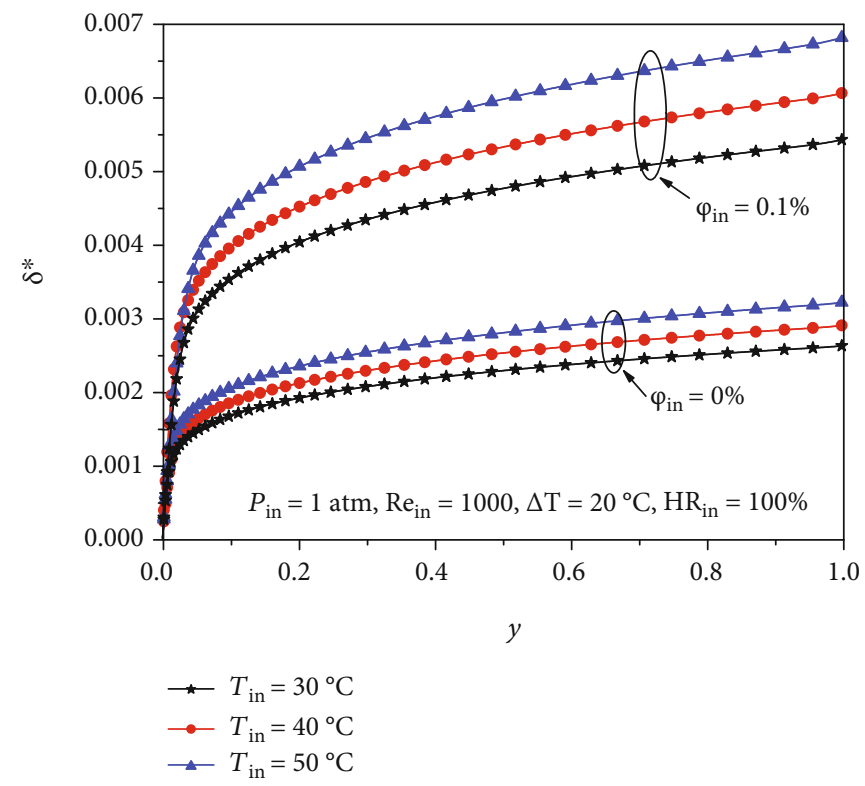

FIGURE 16: Effect of inlet temperature on liquid film thickness.

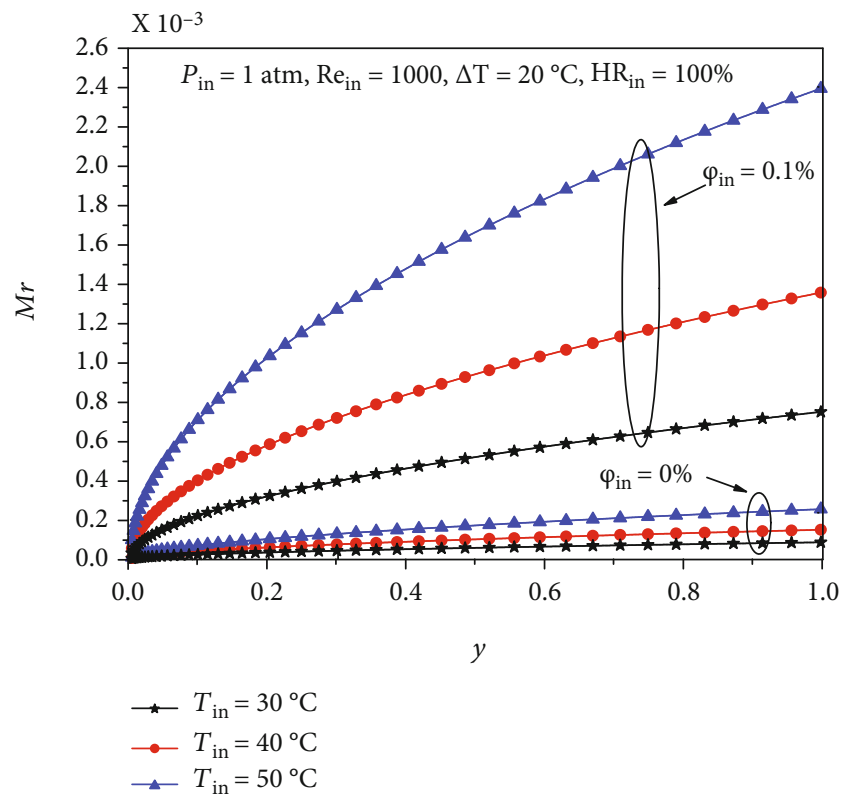

FIGURE 17: Effect of inlet temperature on accumulated condensation rate.

entrance of the channel and decreases significantly as it increases because the mixture cools progressively across the interface during the flow. On the other hand, the profiles of the dimensionless temperature of the mixture are almost parabolic except at the inlet zone, and the maximum temperature is always at the axis of symmetry and decreases along the $\eta$ direction; this decrease is due to the sensible heat transfer to the liquid zone.

Figure 12 shows the bulk concentration profiles $W_{\text {bulk }}$ in both cases with and without nanoparticles. From this figure, we can see that the $W_{\text {bulk }}$ evolution of the mixture shows a strong axial decrease due to the presence of the large concen-

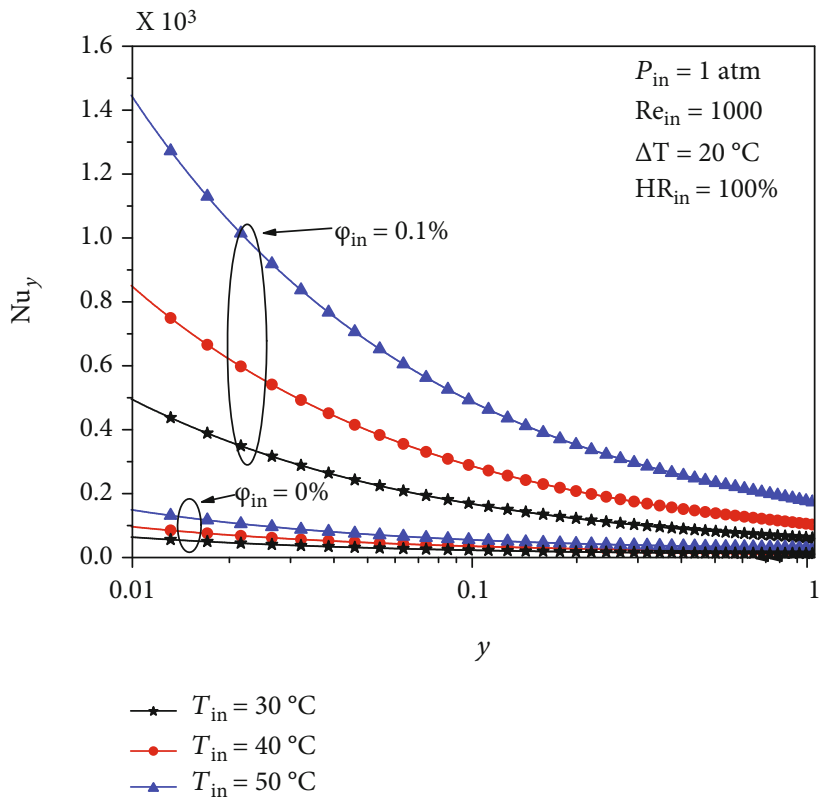

FIGURE 18: Effect of input temperature on the local Nusselt number.

tration gradients in the vicinity of the channel entrance. Moreover, this decrease is due to the loss of the vapor by the condensation process through the interface. The presence of nanoparticles favors the condensation of the vapor.

5.3. Evaluation of Heat and Mass Transfer Parameters on the Process of Nanofluid Condensation. The influence of nanoparticles on the process of condensation depends on the inlet conditions, such as inlet velocity, inlet relative humidity, wall temperature, inlet temperature, and inlet pressure. To distinguish the effect of these parameters, the local Nusselt number, the accumulated condensation rate, and the thickness 


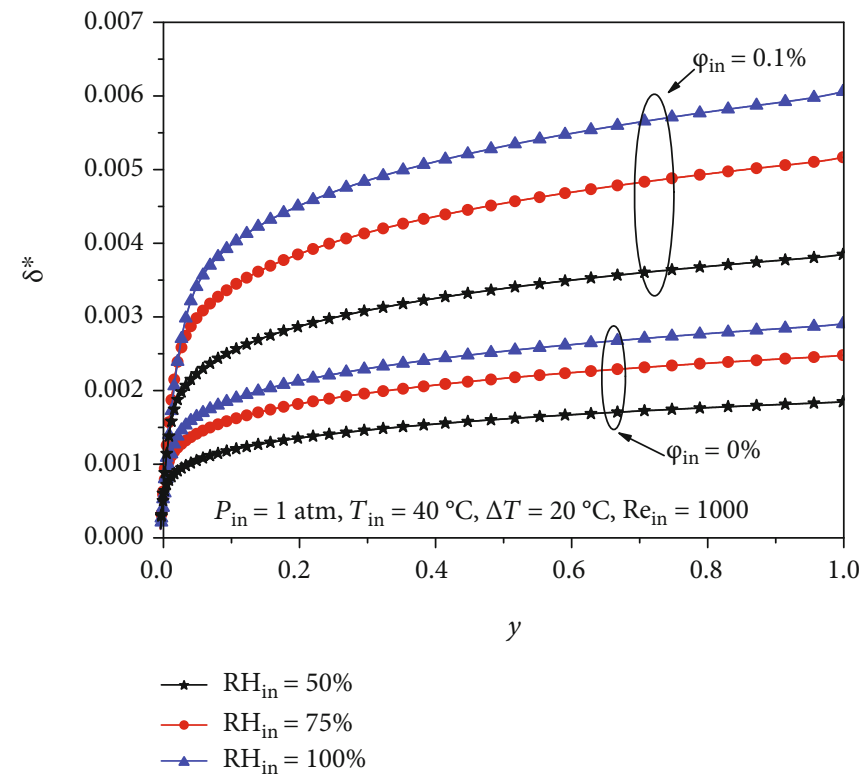

FIGURE 19: Effect of the inlet relative humidity on liquid film thickness.

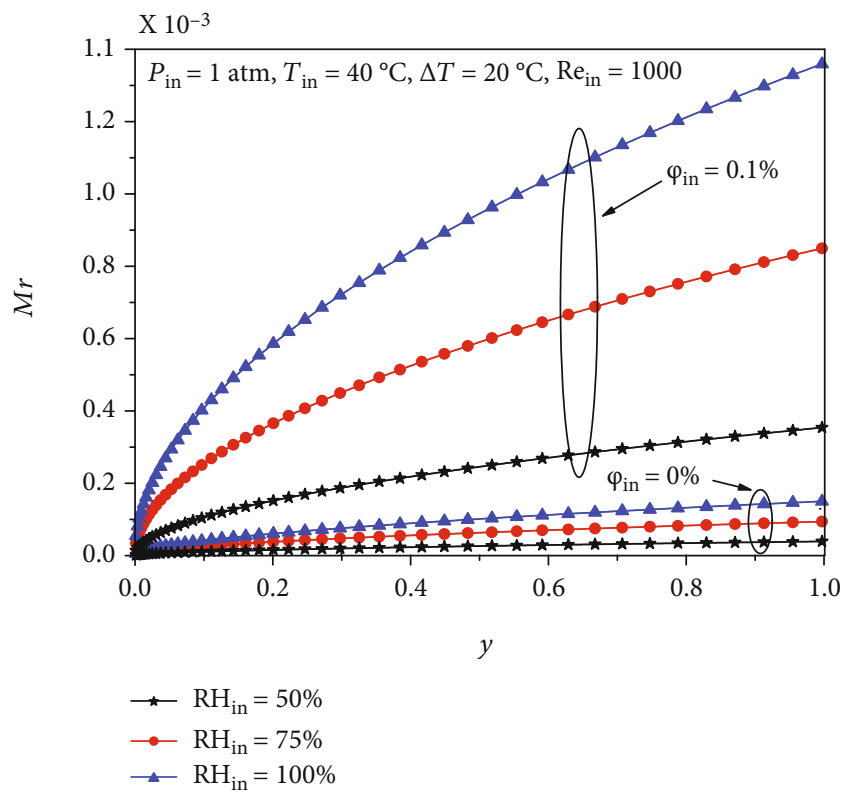

FIGURE 20: Effect of the inlet relative humidity on the accumulated condensation rate.

of the liquid film along the channel are plotted and compared with the case of the pure humid air.

5.3.1. Effect of $\operatorname{Re}_{\text {in }}$ on $\delta^{*}, M r$, and $N u_{y}$. The effect of the Reynolds number is presented in Figures 13-15. In these figures, we have plotted the evolution of the liquid film thickness, the accumulated condensation rate, and the local Nusselt number at the interface along the channel for different Reynolds numbers $\mathrm{Re}_{\text {in }}=500, \mathrm{Re}_{\text {in }}=1000, \mathrm{Re}_{\text {in }}=2000$.

Figure 13 shows that the thickness of the liquid film increases with increasing the Reynolds number; this increase is observed in both cases with and without nanoparticles.

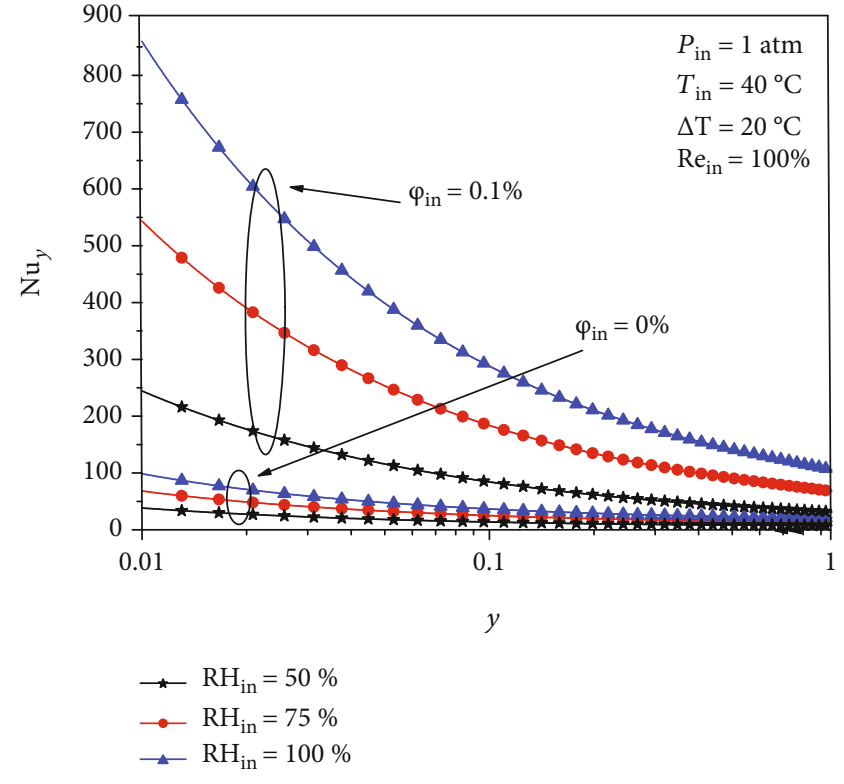

Figure 21: Effect of the inlet relative humidity on the local Nusselt number.

Indeed, the increase in the inlet velocity of the water vapor-air mixture leads to an increase in convective transfers within the channel. The result is a net increase in the flow rate of the interfacial condensate. Comparison of liquid film thickness profiles indicates that the use of nanoparticles improves mass transfer, which promotes condensation. The use of the nanoparticle volume fraction $\varphi=0.1 \%$ leads to an increase in the liquid film thickness of about $\mathrm{R}_{\text {eff }}=2.05$ and $\mathrm{R}_{\text {eff }}=2.12$ for the Reynolds numbers $\mathrm{Re}_{\text {in }}=500$ and $\mathrm{Re}_{\text {in }}=2000$, respectively.

Figure 14 shows that as the Reynolds number increases, the accumulated condensation rate increases in both cases with and without the nanoparticles. The increase in the inlet 


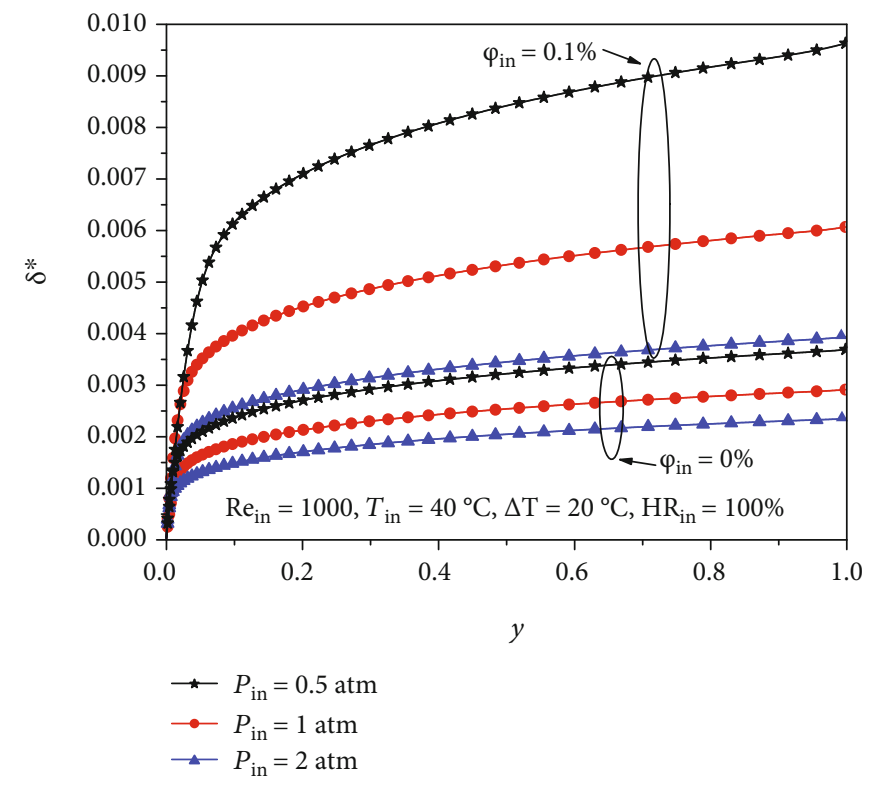

Figure 22: Effect of inlet pressure on liquid film thickness.

Reynolds number leads to an increase in the inlet velocity of the water vapor-air-nanoparticle mixture which leads to an increase in the condensate flow rate across the interface. This results in a clear increase in the accumulated condensation rate obtained by condensation. The addition of $0.1 \%$ of $\mathrm{Cu}$ nanoparticles results in a significant improvement in the amount of condensed steam. In fact, the improvement ratio at the channel outlet is about $R_{\text {eff }}=8.67$ and $R_{\text {eff }}=9.48$, respectively, for the Reynolds numbers $\operatorname{Re}_{\text {in }}=500$ and $\operatorname{Re}_{\text {in }}=2000$.

The effect of the Reynolds number with or without nanoparticles on the Nusselt number is shown in Figure 15. This figure shows that the heat exchanges represented by the Nusselt number decrease along the channel, in both cases with and without nanoparticles, for the three Reynolds numbers studied. This is due to the decrease in the interfacial condensation rate towards the outlet of the channel. As the Reynolds number increases, the effect of the nanoparticles becomes more important. Indeed, by adding a volume fraction of $\varphi$ $=0.1 \%$, we notice that for $\mathrm{Re}_{\text {in }}=500$, the Nusselt number near the inlet is improved by $R_{\text {eff }}=8.33$, by $R_{\text {eff }}=8.61$ for $\mathrm{Re}_{\mathrm{in}}=1000$, and by $R_{\mathrm{eff}}=8.77$ for $\mathrm{Re}_{\mathrm{in}}=2000$.

5.3.2. Effect of $T_{\text {in }}$ on $\delta^{*}, M r$, and $N u_{y}$. The influence of the inlet temperature on the performance of the condensation process of humid air with and without nanoparticles is shown in Figures 16-18.

From Figure 16, we observe that the inlet temperature has a positive effect on the mass transfer and that the condensate film thickness increases by $22.3 \%$ for $\varphi_{\text {in }}=0 \%$ and by $25.5 \%$ for $\varphi_{\text {in }}=0.1 \%$ when the inlet temperature increases from $30^{\circ} \mathrm{C}$ to $50^{\circ} \mathrm{C}$. Indeed, the increase in steam concentration in the inlet region increases the driving force which leads to an increase in the interfacial condensation rate. For an inlet temperature ranging from $30^{\circ} \mathrm{C}$ to $50^{\circ} \mathrm{C}$, the addition of a volume fraction of $0.1 \%$ of $\mathrm{Cu}$ nanoparticles increases the effective improvement ratio by $R_{\text {eff }}=2.06$ and $R_{\text {eff }}=2.11$, respectively.
The amount of steam condensed along the channel, with and without nanoparticles, is significantly improved by increasing the inlet temperature. Figure 17 indicates that increasing inlet temperature results in high interfacial condensation rates. Indeed, when the inlet steam temperature increases and reaches $50^{\circ} \mathrm{C}$, it causes an increase in the inlet concentration of the flow and consequently increases the driving force so that the steam is rapidly condensed; this force is considered a qualitative measure of mass transfer, which leads to an increase in the accumulated condensation rate as well as an increase in the heat flux at the interface. This figure shows that this improvement is more significant by adding a volume fraction $\varphi_{\text {in }}=0.1 \%$ of the nanoparticles. Indeed, the improvement ratio is about $R_{\text {eff }}=8.71$ and $R_{\text {eff }}=9.38$, respectively, when the temperature increases from $30^{\circ} \mathrm{C}$ to $50^{\circ} \mathrm{C}$.

Figure 18 shows the influence of the inlet temperature on the Nusselt number at the interface, for both mixtures (airsteam- $\mathrm{Cu}$ and air-steam). The results show that the improvement of the Nusselt number at the interface is more significant with a high inlet temperature. The presence of the nanoparticles of $\mathrm{Cu}$ improves the physical properties of the base fluid which leads to an improvement of the heat exchange between the mixture flow and the liquid film through the interface. Indeed, the improvement ratio at the channel inlet attains $R_{\text {eff }}=9.53$ for an inlet temperature of $50^{\circ} \mathrm{C}$.

5.3.3. Effect of $R H_{\text {in }}$ on $\delta^{*}, M r$, and $N u_{y}$. The variation of the liquid film thickness, the accumulated condensation rate, and the Nusselt number at the interface, in both cases with and without the nanoparticles and for three input relative humidity values, are shown in Figures 19-21, respectively.

From Figure 19, we notice that the thickness of the condensate film increases on the one hand, with the increase of the inlet relative humidity, and on the other hand, with the addition of a volume fraction $\varphi_{\text {in }}=0.1 \%$ of the $\mathrm{Cu}$ nanoparticles. Increasing the relative inlet humidity increases the 
vapor concentration in the mixture, and consequently, the driving force of condensation increases, which leads to an increase of the mass of condensed vapor. With the addition of nanoparticles, this increase is intensified due to an improvement in the interfacial condensation rate. Figure 19 shows that the presence of nanoparticles increases the thickness of the liquid film at the outlet of the channel by $R_{\text {eff }}=$ 2.09 for a relative humidity of $100 \%$ and by $R_{\text {eff }}=2.08$ for a relative humidity of $50 \%$.

Figure 20 clearly shows that the addition of a volume fraction of $0.1 \%$ of the nanoparticles improves the amount of condensed steam along the channel. Indeed, the improvement rate increases by about $R_{\text {eff }}=9$ and $R_{\text {eff }}=9.08$, respectively, when the humidity increases from $50 \%$ to $100 \%$. Therefore, the improvement ratios obtained from Figures 19 and 20 confirm that the effect of nanoparticles on improving mass transfer is almost the same regardless of the humidity of the inlet flow.

Figure 21 illustrates that the number of Nusselt increases along the channel with increasing relative humidity at the inlet with and without nanoparticles. The addition of nanoparticles improves the local Nusselt number at the interface; this improvement depends on the relative humidity of the inlet flow. Indeed, the improvement ratio of the Nusselt number at the channel inlet increases from $R_{\text {eff }}=6.19$ to $R_{\text {eff }}=8.61$ when the relative humidity increases from $50 \%$ to $100 \%$. Furthermore, according to the definition of the local Nusselt number (equation (33)), the use of nanoparticles causes a higher condensation rate, which leads to a thicker liquid film (Figure 19) and consequently a significant heat transfer to the condensed liquid film. The results confirm that the effect of nanoparticles on improving heat transfer is greater at high relative humidities.

5.3.4. Effect of $P_{\text {in }}$ on $\delta^{*}, M r$, and $N u_{y}$. Figures 22-24 show, respectively, the condensate film thickness, the accumulated condensation rate at the interface, and the local Nusselt number at the interface for the air-steam mixture with and without nanoparticles for different inlet pressures.

The variation of the liquid film thickness for three values of the inlet pressure $\left(P_{\text {in }}=0.5 \mathrm{~atm} ; P_{\text {in }}=1 \mathrm{~atm}\right.$; and $P_{\text {in }}=2$ atm) is shown in Figure 22. When the inlet pressure decreases, the thickness of the liquid condensate with and without nanoparticles increases; this is explained by the increase in the inlet concentration which causes an increase in the driving force. This increase is greater with nanoparticles, so that at an inlet pressure of $P_{\text {in }}=0.5 \mathrm{~atm}$, the thickness of the liquid film improves by about $R_{\text {eff }}=2.61$.

Figure 23 shows that as the inlet pressure decreases, the amount of condensed steam at the interface increases with and without nanoparticles. This increase is more significant with the addition of $0.1 \%$ of $\mathrm{Cu}$ nanoparticles for all inlet pressure values. The effective improvement ratio increases from $R_{\text {eff }}=4.69$ to $R_{\text {eff }}=17.61$ by decreasing the inlet pressure from $P_{\text {in }}=2$ atm to $P_{\text {in }}=0.5$ atm. These results confirm that the effect of nanoparticles on mass transfer improvement is greater at low inlet pressures.

The heat transfer results mainly from the latent heat released by the condensation of the steam. Indeed,

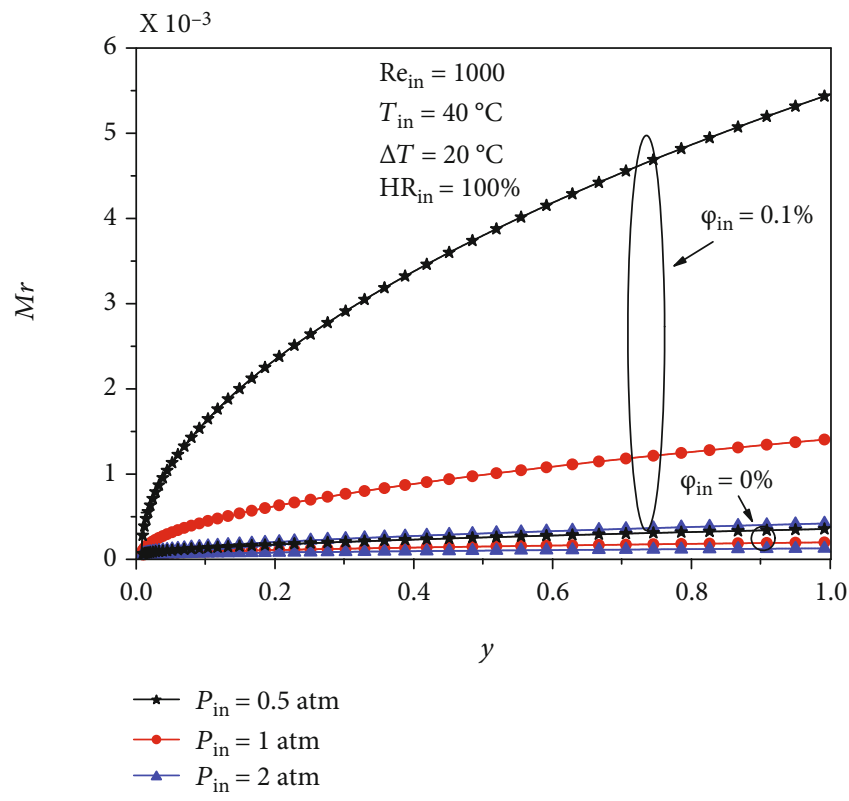

FIGURE 23: Effect of inlet pressure on accumulated condensation rate.

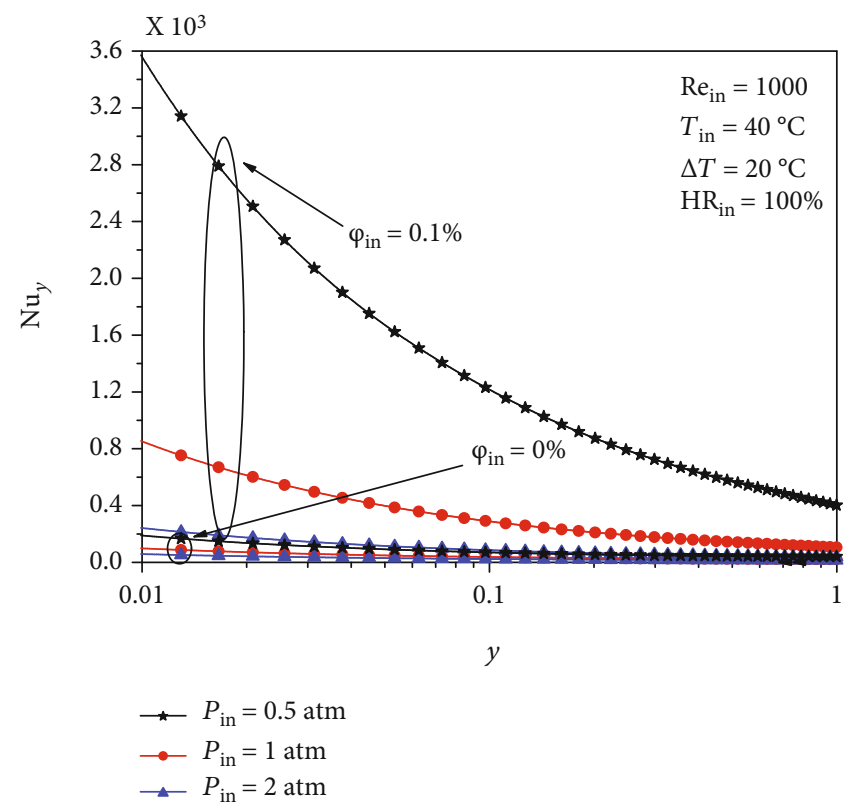

FIgURE 24: Effect of the inlet pressure on the local Nusselt number.

Figures 22 and 23 have shown that the decrease in inlet pressure leads to an increase in the condensate rate at the interface, and consequently, the Nusselt number increases. As shown in Figure 24, the increase in the Nusselt number is more evident with the presence of $\mathrm{Cu}$ nanoparticles which improve the thermophysical properties of the base fluid, leading to the improvement of convective masse transfers with an effective ratio of $R_{\text {eff }}=18.80$ for $P_{\text {in }}=0.5$ atm and $R_{\text {eff }}=4.14$ for $P_{\text {in }}=2 \mathrm{~atm}$. 


\section{Conclusion}

The numerical analysis presented in this article is aimed at studying the condensation of nanofluid film with air as a noncondensable gas through a vertical channel. The effects of adding nanoparticles in the inlet flow on improvement of film condensation and on combined heat and mass transfers were analyzed. The governing equations for the two dimensional, stationary, and laminar flow in both phases have been numerically modeled with different inlet conditions using the finite volume method. Findings of this investigation include the following:

(i) Use of nanoparticles during condensation results in improving the process of heat and mass transfer. The results obtained affirm that the volume fraction of the nanoparticles is an essential factor for the improvement of the thermal and mass transfer compared to the case of the base fluid

(ii) The ratio of heat and mass transfer enhancement in the presence of nanoparticles increases with increasing the Reynolds number $\mathrm{Re}_{\mathrm{in}}$, inlet temperature $T_{\text {in }}$, and inlet relative humidity $\mathrm{RH}_{\text {in }}$ and decreases with increasing inlet pressure $P_{\text {in }}$

(iii) Increase in the volume fraction of the nanofluid further promotes heat and mass transfer. The maximum improvement of $\mathrm{Mr}$ and $\mathrm{Nu}_{y}$ is about $R_{\text {eff }}=9.12$ and $R_{\text {eff }}=4.75$, respectively, with $\varphi_{\text {in }}=$ $0.1 \%$ of $\mathrm{Cu}$ nanoparticles

(iv) Thickness of condensate film in the presence of nanoparticles increases with the increase in $\mathrm{Re}_{\mathrm{in}}$, $T_{\text {in }}$, and $\mathrm{RH}_{\text {in }}$ and with the decrease in $P_{\text {in }}$

(v) Convective heat transfers improved by an effective ratio of $R_{\text {eff }}=18.80$ at $P_{\text {in }}=0.5 \mathrm{~atm}$ and by $R_{\text {eff }}=$ 4.14 at $P_{\text {in }}=2$ atm.

The phenomenon of condensation of humid air is a process often experienced in many industrial installations. Hence, providing more effort to better comprehend the mechanisms of this phase change process is important. According to the results of this study, the use of suspended nanoparticles improves heat and mass transfer processes during condensation, and the results detailed above could be useful in finding the optimal conditions for using nanoparticles to improve heat and mass transfer during condensation processes.

\section{Nomenclature}

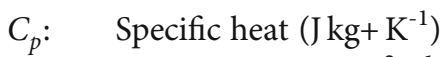

$D: \quad$ Mass diffusivity $\left(\mathrm{m}^{2} \mathrm{~s}^{-1}\right)$

$d$ : $\quad$ Moisture content $(\mathrm{kg} / \mathrm{kg}$ of dry air)

$g: \quad$ Gravitational acceleration $\left(\mathrm{m} \mathrm{s}^{-2}\right)$

$H$ : $\quad$ Half of plate spacing $(\mathrm{m})$

$h_{f g}: \quad$ Latent heat $\left(\mathrm{J} \mathrm{kg}^{-1}\right)$

$J^{\prime \prime}$ : Condensation mass flux $\left(\mathrm{kg} \mathrm{m}^{-2} \mathrm{~s}^{-1}\right)$

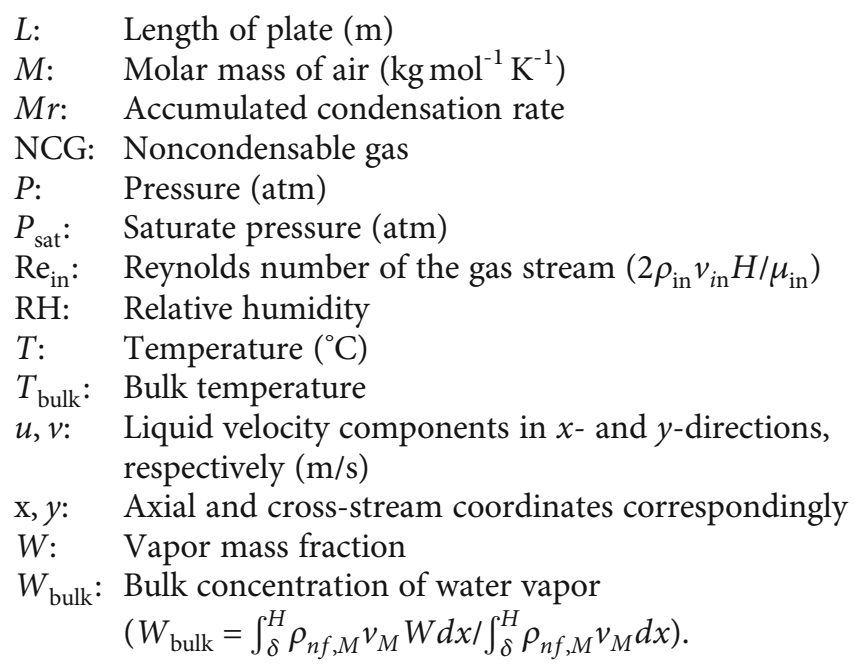

Greek Symbols

$\rho: \quad$ Density $\left(\mathrm{kg} \mathrm{m}^{-3}\right)$

$\mu: \quad$ Dynamic viscosity $\left(\mathrm{kg} \mathrm{m}^{-1} \mathrm{~s}^{-1}\right)$

$\lambda$ : Thermal conductivity $\left(\mathrm{W} \mathrm{m}^{-1} \mathrm{~K}^{-1}\right)$

$\delta: \quad$ Condensate layer thickness $(\mathrm{m})$

$\delta^{*}: \quad$ Dimensionless condensate layer thickness $(\delta / H)$

$\delta^{* *}:$ Dimensionless condensate layer thickness $(\delta / 2 H)$

$\chi, \eta$ : Axial and cross-stream transformed coordinates, respectively.

\section{Subscripts}

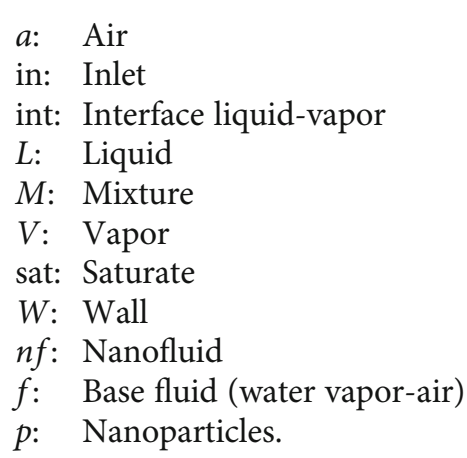

\section{Data Availability}

The data used to support the findings of this study are included within the article.

\section{Conflicts of Interest}

The authors declare that they have no conflicts of interest.

\section{References}

[1] I. Z. Famileh, J. A. Esfahani, and K. Vafai, "Effect of nanoparticles on condensation of humid air in vertical channels," International Journal of Thermal Sciences, vol. 112, pp. 470483, 2017.

[2] O. Mahian, H. Oztop, I. Pop, S. Mahmud, and S. Wongwises, "Entropy generation between two vertical cylinders in the presence of MHD flow subjected to constant wall 
temperature," International Communications in Heat and Mass Transfer, vol. 44, pp. 87-92, 2013.

[3] K. al-Salem, H. F. ztop, I. Pop, and Y. Varol, "Effects of moving lid direction on MHD mixed convection in a linearly heated cavity," International Journal of Heat and Mass Transfer, vol. 55, no. 4, pp. 1103-1112, 2012.

[4] H. F. Oztop, M. Mobedi, E. Abu-Nada, and I. Pop, “A heatline analysis of natural convection in a square inclined enclosure filled with a $\mathrm{CuO}$ nanofluid under non-uniform wall heating condition," International Journal of Heat and Mass Transfer, vol. 55, no. 19-20, pp. 5076-5086, 2012.

[5] M. R. Shirkhani, A. Hoshyar, and D. D. Ganji, “Application of VIM, HPM and CM to the system of strongly nonlinear fin problem," Journal of Engineering and Technology (JET), vol. 9, no. 2, 2018.

[6] M. M. Peiravi, J. Alinejad, D. D. Ganji, and S. Maddah, “3D optimization of baffle arrangement in a multi-phase nanofluid natural convection based on numerical simulation," International Journal of Numerical Methods for Heat \& Fluid Flow, vol. 30, no. 5, pp. 2583-2605, 2019.

[7] M. M. Peiravi, J. Alinejad, D. Ganji, and S. Maddah, "Numerical study of fins arrangement and nanofluids effects on threedimensional natural convection in the cubical enclosure," Transport Phenomena in Nano and Micro Scales, vol. 7, no. 2, pp. 97-112, 2019.

[8] W. Nusselt, "The condensation of steam on cooled surfaces," Zeitschrift des Vereins Deutscher Ingenieure, vol. 60, pp. 541546, 1916.

[9] V. Srzic, H. M. Soliman, and S. J. Ormiston, "Analysis of laminar mixed-convection condensation on isothermal plates using the full boundary-layer equations: mixtures of a vapor and a lighter gas," International Journal of Heat and Mass Transfer, vol. 42, no. 4, pp. 685-695, 1999.

[10] Z. A. Hammou, B. Benhamou, N. Galanis, and J. Orfi, "Laminar mixed convection of humid air in a vertical channel with evaporation or condensation at the wall," International Journal of Thermal Sciences, vol. 43, no. 6, pp. 531-539, 2004.

[11] N. K. Maheshwari, D. Saha, R. K. Sinha, and M. Aritomi, "Investigation on condensation in presence of a noncondensable gas for a wide range of Reynolds number," Nuclear Engineering and Design, vol. 227, no. 2, pp. 219-238, 2004.

[12] A. Belhadj Mohamed, J. Orfi, C. Debissi, and S. Ben Nasrallah, "Condensation of water vapor in a vertical channel by mixed convection of humid air in the presence of a liquid film flowing down,” Desalination, vol. 204, no. 1-3, pp. 471-481, 2007.

[13] A. Giri, D. Bhuyan, and B. Das, "A study of mixed convection heat transfer with condensation from a parallel plate channel," International Journal of Thermal Sciences, vol. 98, pp. 165-178, 2015.

[14] J. D. Li, "CFD simulation of water vapour condensation in the presence of non-condensable gas in vertical cylindrical condensers," International Journal of Heat and Mass Transfer, vol. 57, no. 2, pp. 708-721, 2013.

[15] G. Caruso, D. Vitale di Maio, and A. Naviglio, "Condensation heat transfer coefficient with noncondensable gases inside near horizontal tubes," Desalination, vol. 309, pp. 247-253, 2013.

[16] G. Caruso and D. Vitale di Maio, "Heat and mass transfer analogy applied to condensation in the presence of noncondensable gases inside inclined tubes," International Journal of Heat and Mass Transfer, vol. 68, pp. 401-414, 2014.
[17] G. Zschaeck, T. Frank, and A. D. Burns, "CFD modelling and validation of wall condensation in the presence of non- condensable gases," Nuclear Engineering and Design, vol. 279, pp. 137-146, 2014.

[18] D. Hammoudi, A. Benabdesselam, A. Azzi, and M. A. Kassim, "Numerical modeling of steam condensation in vertical channel in presence of noncondensable gas," International Journal of Thermal Sciences, vol. 126, pp. 263-271, 2018.

[19] M. Ait Hssain, Y. el Hammami, R. Mir, S. Armou, and K. ZineDine, "Numerical analysis of laminar convective condensation with the presence of noncondensable gas flowing downward in a vertical channel," Mathematical Problems in Engineering, vol. 2019, Article ID 7941363, 15 pages, 2019.

[20] X. Wu, F. Chu, Y. Zhu, and Q. Li, "Vapor free convection film condensation heat transfer in the presence of non- condensable gases with smaller molecular weights than the vapor," Applied Thermal Engineering, vol. 130, pp. 1611$1618,2018$.

[21] A. A. Avramenko, I. V. Shevchuk, A. I. Tyrinov, and D. G. Blinov, "Heat transfer at film condensation of stationary vapor with nanoparticles near a vertical plate," Applied Thermal Engineering, vol. 73, no. 1, pp. 391-398, 2014.

[22] A. A. Avramenko, I. V. Shevchuk, A. I. Tyrinov, and D. G. Blinov, "Heat transfer at film condensation of moving vapor with nanoparticles over a flat surface," International Journal of Heat and Mass Transfer, vol. 82, pp. 316-324, 2015.

[23] M. Turkyilmazoglu, "Analytical solutions of single and multiphase models for the condensation of nanofluid film flow and heat transfer," European Journal of Mechanics - B/Fluids, vol. 53, pp. 272-277, 2015.

[24] H. el Mghari, H. Louahlia-Gualous, and E. Lepinasse, "Numerical study of nanofluid condensation heat transfer in a square microchannel," Numerical Heat Transfer, Part A: Applications, vol. 68, no. 11, pp. 1242-1265, 2015.

[25] A. Malvandi, D. D. Ganji, and I. Pop, "Laminar filmwise condensation of nanofluids over a vertical plate considering nanoparticles migration," Applied Thermal Engineering, vol. 100, pp. 979-986, 2016.

[26] M. Hatami, S. Mosayebidorcheh, and D. Jing, "Two-phase nanofluid condensation and heat transfer modeling using least square method (LSM) for industrial applications," Heat and Mass Transfer, vol. 53, no. 6, pp. 2061-2072, 2017.

[27] Q. Peng, L. Jia, C. Dang, X. Zhang, and Q. Huang, "Experimental investigation on flow condensation of R141b with $\mathrm{CuO}$ nanoparticles in a vertical circular tube," Applied Thermal Engineering, vol. 129, pp. 812-821, 2018.

[28] F. Hassaninejadfarahani, M. K. Guyot, and S. J. Ormiston, "Numerical analysis of mixed-convection laminar film condensation from high air mass fraction steam-air mixtures in vertical tubes," International Journal of Heat and Mass Transfer, vol. 78, pp. 170-180, 2014.

[29] E. C. Siow, S. J. Ormiston, and H. M. Soliman, “A two-phase model for laminar film condensation from steam-air mixtures in vertical parallel-plate channels," Heat and Mass Transfer, vol. 40, no. 5, pp. 365-375, 2004.

[30] H. F. Oztop and E. Abu-Nada, "Numerical study of natural convection in partially heated rectangular enclosures filled with nanofluids," International Journal of Heat and Fluid Flow, vol. 29, no. 5, pp. 1326-1336, 2008.

[31] X. Zhang, H. Gu, and M. Fujii, "Effective thermal conductivity and thermal diffusivity of nanofluids containing spherical and 
cylindrical nanoparticles," Journal of Applied Physics, vol. 100, no. 4, article 044325, 2006.

[32] H. C. Brinkman, "The viscosity of concentrated suspensions and solutions," The Journal of Chemical Physics, vol. 20, no. 4, pp. 571-571, 1952.

[33] J. C. Maxwell, A Treatise on Electricity and Magnetism: pt. III. Magnetism. pt. IV. Electromagnetism (Vol. 2), Clarendon press, 1881.

[34] S. V. Patankar, Numerical heat transfer and fluid flow, Hemisphere Publ. Corp., New York, 1980.

[35] P. D. Lebedev, A. M. Baklastov, and Z. F. Sergazin, "Aerodynamics, heat and mass transfer in vapour condensation from humid air on a flat plate in a longitudinal flow in asymmetrically cooled slot," International Journal of Heat and Mass Transfer, vol. 12, no. 8, pp. 833-841, 1969. 